\title{
Review of Robust Data Exchange Using Optical Nonlinearities
}

\author{
Jian Wang ${ }^{1,2}$ and Alan E. Willner ${ }^{2}$ \\ ${ }^{1}$ Wuhan National Laboratory for Optoelectronics, College of Optoelectronic Science and Engineering, Huazhong University of Science \\ and Technology, Hubei, Wuhan 430074, China \\ ${ }^{2}$ Department of Electrical Engineering, University of Southern California, Los Angeles, CA 90089, USA
}

Correspondence should be addressed to Jian Wang, jwang@mail.hust.edu.cn

Received 13 July 2011; Accepted 4 November 2011

Academic Editor: Giulio Cerullo

Copyright ( 2012 J. Wang and A. E. Willner. This is an open access article distributed under the Creative Commons Attribution License, which permits unrestricted use, distribution, and reproduction in any medium, provided the original work is properly cited.

\begin{abstract}
Data exchange, namely bidirectional information swapping, provides enhanced flexibility compared to the unidirectional information transfer. To fulfill the rapid development of high-speed large-capacity optical communications with emerging multiplexing/demultiplexing techniques and advanced modulation formats, a laudable goal would be to achieve data exchange in different degrees of freedom (wavelength, time, polarization), for different modulation formats (OOK, DPSK, DQPSK, polmuxed), and at different granularities (entire data, groups of bits, tributary channels). Optical nonlinearities are potentially suitable candidates to enable data exchange in the wavelength, time, and polarization domains. In this paper, we will review our recent works towards robust data exchange by exploiting miscellaneous optical nonlinearities, including the use of cSFG/DFG in a PPLN waveguide for time- (groups of bits) and channel-selective data exchange and tributary channel exchange between two WDM+OTDM signals, nondegenerate FWM in an HNLF for phase-transparent data exchange (DPSK, DQPSK), bidirectional degenerate FWM in an HNLF for multi-channel data exchange, and Kerr-induced nonlinear polarization rotation in an HNLF for tributary channel exchange of a pol-muxed DPSK OTDM signal. The demonstrated data exchanges in different degrees of freedom, for different modulation formats, and at different granularities, open the door for alternative approaches to achieve superior network performance.
\end{abstract}

\section{Introduction}

The rapid growth in network capacity and traffic rates raises the significance of data traffic grooming, which is considered to be a promising technique for enhancing the efficiency and flexibility of networks $[1,2]$. Data grooming techniques can take many forms in wavelength, time, and polarization domains, such as tunable optical delay for buffering, add/drop, (de)multiplexing, multicasting, and data exchange [3-17]. Data exchange is an important concept for efficiently utilizing network resources and improving network performance. Generally speaking, data exchange refers to the information swapping between different wavelengths/time slots/polarizations. In the wavelength domain (e.g., wavelength-division multiplexed (WDM) network), data exchange, which is also known as wavelength interchange or wavelength exchange, would require the swapping of data from one wavelength with the data from another wavelength [17]. Extensions of data exchange would expect the data swapping between different time slots in the time domain (e.g., optical timedivision multiplexed (OTDM) network) or different polarization states in the polarization domain (e.g., polarizationmultiplexed (pol-muxed) network). Moreover, the recently increasing interest of advanced modulation formats would require the data exchange to be available for different modulation formats, such as on-off keying (OOK), differential phase-shift keying (DPSK), differential quadrature phaseshift keying (DQPSK), and pol-muxed $[18,19]$.

Optical nonlinearities (e.g., $\chi^{(2)}$ and $\chi^{(3)}$ ) [20], including cascaded sum- and difference-frequency generation (cSFG/DFG), degenerate/nondegenerate four-wave mixing (FWM), and Kerr-induced nonlinear polarization rotation, are potentially suitable candidates to enable data exchange. Previous reports of data exchange include the use of degenerate FWM in an optical parametric loop mirror (PALM) [21, 22], nondegenerate FWM in a highly nonlinear fiber (HNLF) 


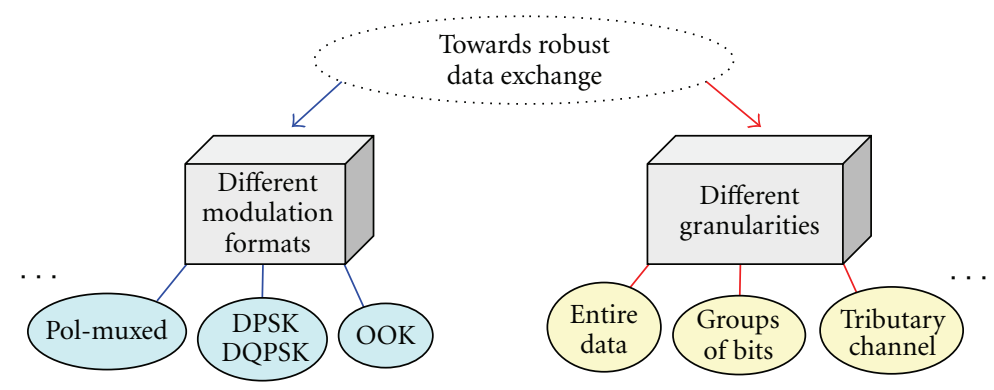

FIgURE 1: Schematic illustration of robust data exchange

[23-31], and second-order nonlinearity in a two-dimensional nonlinear photonic crystal $[32,33]$. For example, PALMbased wavelength interchange for OOK [21] and $10 \mathrm{Gbit} / \mathrm{s}$ DPSK signals [22], nondegenerate FWM-based wavelength exchange for $10 \mathrm{Gbit} / \mathrm{s}$ non-return-to-zero (NRZ) signals [26], byte-level exchange for $10 \mathrm{Gbit} / \mathrm{s}$ return-to-zero (RZ) signals $[28,29]$, and pulsed pump exchange for tributary demultiplexing $[30,31]$ have been demonstrated, showing impressive performance.

In this paper, we will review our recent works towards robust data exchange using optical nonlinearities. As shown in Figure 1, a laudable goal would be to achieve data exchange for different modulation formats (OOK, DPSK, DQPSK, pol-muxed) and at different granularities (entire data, groups of bits, tributary channels). Four kinds of optical nonlinearities are employed: (1) cSFG/DFG in a periodically poled lithium niobate (PPLN) waveguide [34-38]; (2) nondegenerate FWM in an HNLF [39-42]; (3) bidirectional degenerate FWM in an HNLF [43-46]; (4) Kerr-induced nonlinear polarization rotation in an HNLF $[47,48]$. First, we present the theoretical analyses of PPLN-based data exchange. Analytical solutions are derived to indicate the exchange condition [34]. We propose a time- (groups of bits) and channel-selective data exchange between WDM channels. $40 \mathrm{Gbit} / \mathrm{s}$ channel-selective data exchange between four WDM channels is demonstrated with a power penalty of $\sim 4 \mathrm{~dB}$ at a bit-error rate (BER) of $10^{-9}[35,36]$. We further propose and demonstrate PPLN-based data exchange in both wavelength and time domains at a fine granularity, that is, low-speed (10 Gbit/s) tributary channel exchange between two WDM high-speed (160 Gbit/s) OTDM signals. Less than $4 \mathrm{~dB}$ power penalty is observed at a BER of $10^{-9}$ $[37,38]$. Second, we report the modulation-format-transparency characteristic of the data exchange based on nondegenerate FWM. We demonstrate phase-transparent data exchange between two $40 \mathrm{Gbit} / \mathrm{s}$ NRZ-DPSK signals with a power penalty of $\sim 4.5 \mathrm{~dB}$ at a BER of $10^{-9}[39,40]$. Data exchange of $100 \mathrm{Gbit} / \mathrm{s}$ RZ-DQPSK signals is further demonstrated with a power penalty of less than $5 \mathrm{~dB}$ at a BER of $10^{-9}$ $[41,42]$. Third, we propose a simple approach to performing simultaneous multichannel data exchange using bidirectional degenerate FWM in an HNLF. Simultaneous ITUgrid-compatible four-channel $100 \mathrm{Gbit} / \mathrm{s}$ RZ-DQPSK data exchange is demonstrated with a power penalty of less than $4.7 \mathrm{~dB}$ at a BER of $10^{-9}[43,44]$. Moreover, we build a recon- figurable Tbit/s network switching element by use of doublepass liquid crystal on silicon (LCoS) technology assisted by bidirectional degenerate FWM in a single HNLF. We achieve a $2.3 \mathrm{Tbit} / \mathrm{s}$ (23-channel $100 \mathrm{Gbit} / \mathrm{s} \mathrm{RZ}-\mathrm{DQPSK}$ ) multifunctional grooming switch, performing simultaneous selective add/drop, switchable data exchange, and power equalization. Power penalties less than $1.2 \mathrm{~dB}$ for two-channel add, $0.5 \mathrm{~dB}$ for two-channel drop, and $5 \mathrm{~dB}$ for six-channel data exchange are observed at a BER of $10^{-9}[45,46]$. Finally, using Kerr effect-induced nonlinear birefringence in an HNLF, we demonstrate $10 \mathrm{Gbit} / \mathrm{s}$ tributary channel exchange between two orthogonal polarizations of a $160 \mathrm{Gbit} / \mathrm{s}$ polmuxed DPSK OTDM signal with a power penalty of less than $4 \mathrm{~dB}$ at a BER of $10^{-9}[47,48]$.

\section{Concept of Data Exchange}

Robust data exchange in the wavelength, time, and polarization domains might be valuable for superior network performance [49]. As an example, a desirable goal of data exchange would be to efficiently utilize nonlinearities in the wavelength domain, such that the data between two different wavelengths can be "exchanged," that is, swapped, using single nonlinear processes in a single device. Figure 2(a) illustrates the basic concept of data exchange in the wavelength domain (known as wavelength interchange or wavelength exchange) $[21,25]$, which is a wavelength-domain data manipulation enabling the swapping of data between two different wavelengths. One straightforward way, as shown in Figure 2(b), is to use two separate wavelength converters (WCs) with one performing the wavelength conversion from signal A to signal B and the other from signal B to signal A. Towards single-device operation, one simple way of data exchange in the wavelength domain is to explore the combined signal depletion and wavelength conversion effects in a nonlinear device including a piece of HNLF or a PPLN waveguide. Nondegenerate FWM $\left(\chi^{(3)}\right)$ in an HNLF [23-31, 39-42] and cascaded second-order nonlinearities $\left(\chi^{(2)}: \chi^{(2)}\right)$ in a PPLN waveguide [34-38] are potential choices to realize such data exchange. As shown in Figure 2(c), due to the signal depletion and wavelength conversion effects, the data carried by signal $\mathrm{A}$ is consumed and converted to the wavelength of signal $\mathrm{B}$ and vice versa. This enables single-device-based data exchange in the wavelength domain. Similar concepts of data 


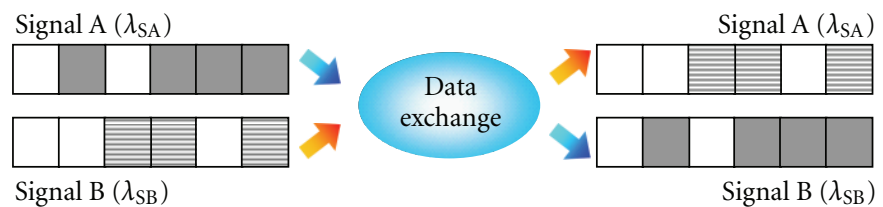

(a)

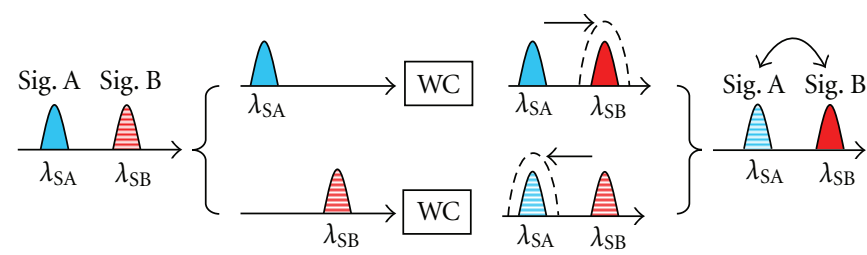

(b)

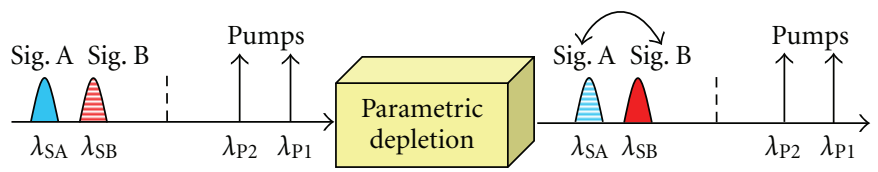

(c)

FIGURE 2: (a) Concept of data exchange in the wavelength domain (wavelength interchange/exchange). (b) Data exchange by two separate wavelength converters. (c) An example of data exchange by signal depletion and wavelength conversion in a single nonlinear device.

exchange in the time and polarization domains, enabled by various optical nonlinearities, are also available, which will be discussed with more details in the following sections.

\section{Recent Advances for Robust Data Exchange Using Optical Nonlinearities}

3.1. Data Exchange Using cSFG/DFG in a Single PPLN Waveguide [34-38]. As depicted in Figure 2(c), data exchange based on signal depletion and wavelength conversion of cSFG/DFG involves two signals and two pumps, which can be described by the coupled-mode equations. To better understand the single-PPLN-based data exchange, under the slowly varying amplitude approximation, we can derive the following analytical solutions to the complex amplitudes of signal A $\left(A_{\mathrm{SA}}(L)\right)$ and signal $\mathrm{B}\left(A_{\mathrm{SB}}(L)\right)$ after data exchange [34]:

$$
\begin{aligned}
A_{\mathrm{SA}}(L)= & A_{\mathrm{SA}}(0)+\omega_{\mathrm{SA}} \omega_{\mathrm{SF}} \kappa_{1} \frac{1}{M^{2}} A_{\mathrm{P} 1}^{*}(0) \\
& {\left[\kappa_{1} A_{\mathrm{P} 1}(0) A_{\mathrm{SA}}(0)+\kappa_{2} A_{\mathrm{P} 2}(0) A_{\mathrm{SB}}(0)\right] } \\
& \times[\cos (M L)-1], \\
A_{\mathrm{SB}}(L)= & A_{\mathrm{SB}}(0)+\omega_{\mathrm{SB}} \omega_{\mathrm{SF}} \kappa_{2} \frac{1}{M^{2}} A_{\mathrm{P} 2}^{*}(0) \\
& \times\left[\kappa_{1} A_{\mathrm{P} 1}(0) A_{\mathrm{SA}}(0)+\kappa_{2} A_{\mathrm{P} 2}(0) A_{\mathrm{SB}}(0)\right] \\
& \times[\cos (M L)-1],
\end{aligned}
$$

where $M=\sqrt{\omega_{\mathrm{SA}} \omega_{\mathrm{SF}} \kappa_{1}^{2} P_{\mathrm{P} 1}(0)+\omega_{\mathrm{SB}} \omega_{\mathrm{SF}} \kappa_{2}^{2} P_{\mathrm{P} 2}(0)} \cdot A_{\mathrm{SA}}(0)$, $A_{\mathrm{SB}}(0), A_{\mathrm{P} 1}(0)$, and $A_{\mathrm{P} 2}(0)$ are the input complex amplitudes of signal A, signal B, pump 1, and pump 2, respectively.
$P_{\mathrm{P} 1}(0)$ and $P_{\mathrm{P} 2}(0)$ are the input power of pump 1, and pump 2. $\kappa_{1}\left(\kappa_{2}\right)$ refers to the coupling coefficient of the secondorder nonlinear interaction involving signal A (signal B) and pump 1 (pump 2). $\omega_{\mathrm{SA}}, \omega_{\mathrm{SB}}$, and $\omega_{\mathrm{SF}}$ are the angular frequencies of signal A, signal B, and sum-frequency (SF) wave, respectively. $L$ is the waveguide length.

When ignoring the initial pump phase and setting the same power for two input pumps, we can further simplify (1a) and (1b) as follows:

$$
\begin{aligned}
& A_{\mathrm{SA}}(L)=\frac{\cos (M L)+1}{2} A_{\mathrm{SA}}(0)+\frac{\cos (M L)-1}{2} A_{\mathrm{SB}}(0), \\
& A_{\mathrm{SB}}(L)=\frac{\cos (M L)-1}{2} A_{\mathrm{SA}}(0)+\frac{\cos (M L)+1}{2} A_{\mathrm{SB}}(0) .
\end{aligned}
$$

When satisfying the following relationship written by

$$
M z=(2 N+1) \pi, \quad N=0,1,2,3 \ldots,
$$

we can obtain

$$
A_{\mathrm{SA}}(L)=-A_{\mathrm{SB}}(0), \quad A_{\mathrm{SB}}(L)=-A_{\mathrm{SA}}(0) .
$$

From (4) it can be clearly seen that data exchange between signal A and signal B is achieved under the exchange condition governed by (3). In particular, beyond the data exchange for OOK signal, the complex relationship in (4) also implies the modulation-format-transparency characteristic of PPLN-based data exchange. Figure 3 shows an example of simulation results for $40 \mathrm{Gbit} / \mathrm{s}$ data exchange based on signal depletion and wavelength conversion of cSFG/DFG in a single PPLN waveguide [34]. It is shown that the data information carried by signal A and signal B is swapped after data exchange. 


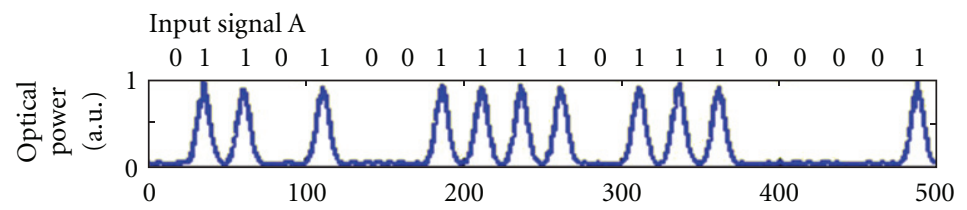

(a)

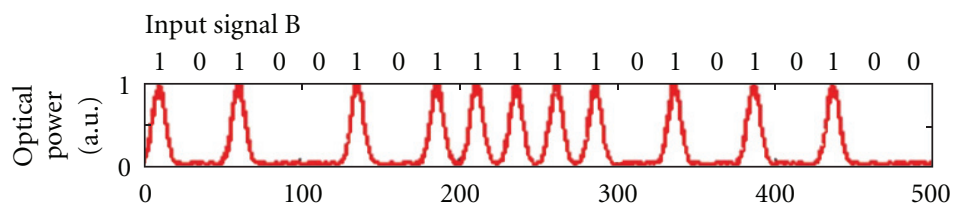

(b)

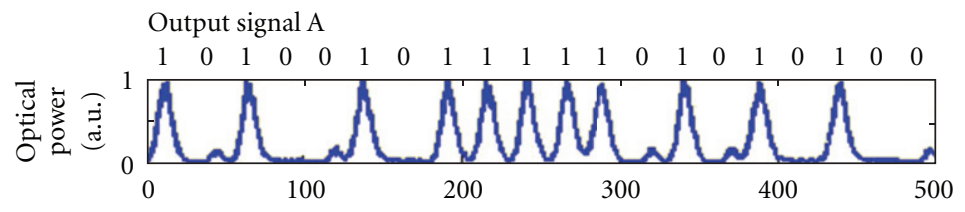

(c)

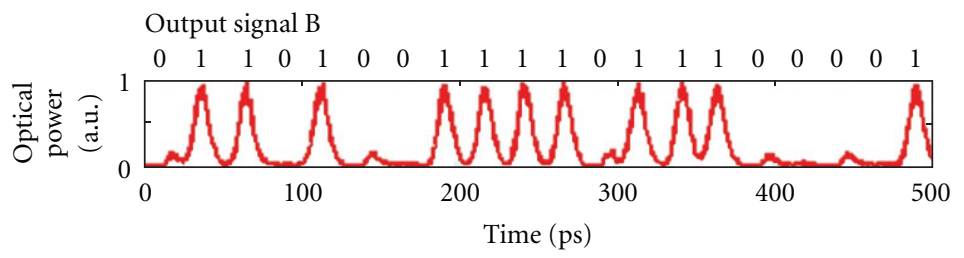

(d)

FIgURE 3: Simulation results of single-PPLN-based $40 \mathrm{Gbit} / \mathrm{s}$ data exchange.

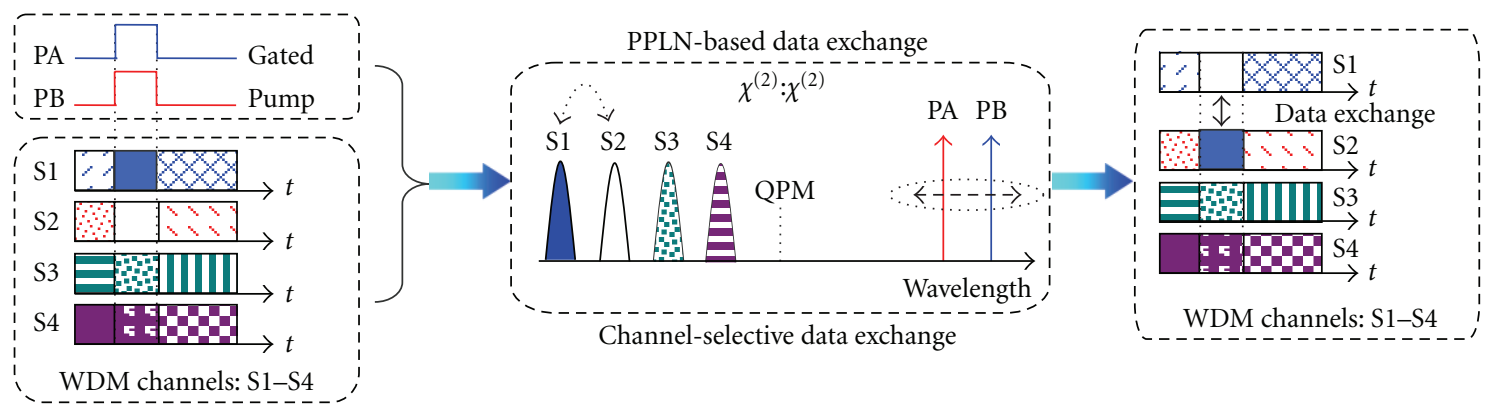

FIgURE 4: Concept of single-PPLN-based time- and channel-selective data exchange between WDM channels.

Following the similar principle of PPLN-based data exchange using signal depletion and wavelength conversion of cSFG/DFG, we can further perform robust data exchange functions, including time- and channel-selective data exchange between WDM channels $[35,36]$ and low-speed tributary channel exchange of high-speed OTDM signals [37, 38].

The conceptual diagram of the proposed single-PPLNbased time- and channel-selective data exchange between WDM channels is illustrated in Figure 4 [35, 36]. Multiple WDM channels (S1-S4) and two synchronized gated pumps (PA, PB) are coupled into a PPLN waveguide, in which cSFG/DFG processes take place. The wavelength selectivity of the quasi-phase matching (QPM) condition allows selection of channels for data exchange by proper choice of the two pump wavelengths. For proper QPM of both cSFG/DFG processes, the two pump wavelengths are nearly symmetric to the two exchanged data wavelengths with respect to the QPM wavelength. For instance, as illustrated in Figure 4, within the gated pump pulse duration, PB mixes with S1 to produce an SF wave through the SFG process. Meanwhile, the SF wave interacts with PA to generate a new idler at the wavelength of S2 by the subsequent DFG process. During such nonlinear interactions, S1 can be depleted [50-52] and converted to S2 by means of proper control of the pump powers. Similarly, PA and S2 participate in the SFG process to create an SF wave, which simultaneously interacts with $\mathrm{PB}$ to yield an idler at the wavelength of S1 via the DFG process. Thus, S2 can also be consumed with its data copied onto S1. 


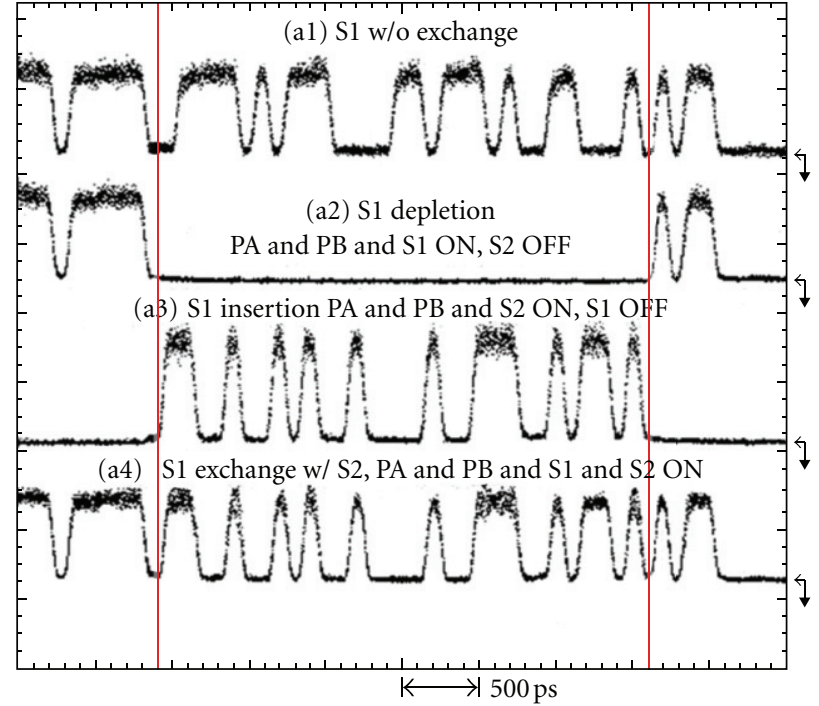

(a5) S1 w/o exchange

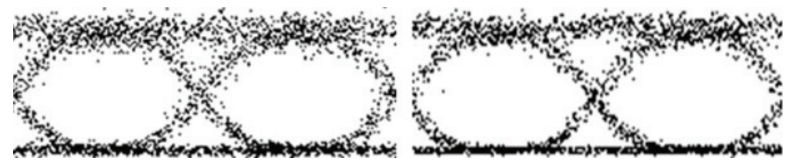

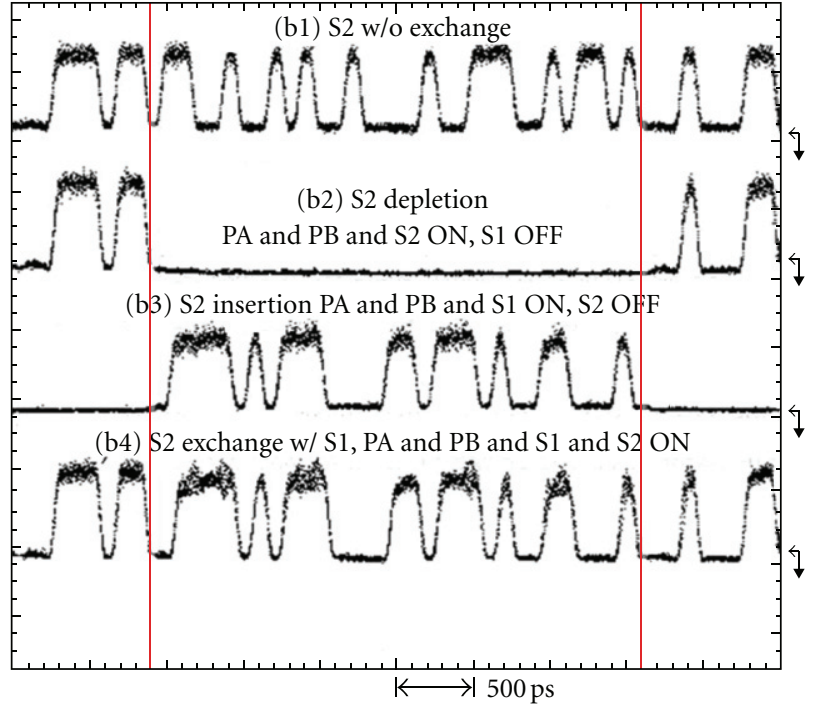

(b5) S2 w/o exchange

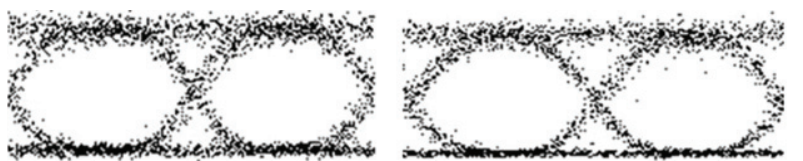

Figure 5: Measured (a1-a4) and (b1-b4) temporal waveforms and (a5), (a6), (b5), and (b6) eye diagrams of 10 Gbit/s data exchange.

Consequently, it is expected to implement optical data exchange between S1 and S2 without the use of additional spectrum and touching other channels. Note that time- and channel-selective data exchange in specific time slots and between selective WDM channels can be accomplished by appropriately choosing the gated pump pulse duration and adjusting the pump wavelengths.

We first demonstrate the data exchange between two $10 \mathrm{Gbit} / \mathrm{s}$ signals. Two gated pumps with a duty cycle of $1 / 127$ and a pulse duration of $\sim 3.2 \mathrm{~ns}$ are employed. The average power of each signal and peak power of each pump coupled into the PPLN waveguide are about $4 \mathrm{~mW}$ and $1 \mathrm{~W}$, respectively. Figure 5 displays the observed temporal waveforms and eye diagrams of data exchange. The time slots between the two straight lines correspond to the gated pump pulse duration, in which data exchange occurs. When S1 and the two pumps are on while S2 is off, the data of S1 within the gated pump pulse duration is depleted (a2) and converted to the wavelength of S2 (b3). Similarly, we can also observe the depletion of S2 (b2) and the conversion from S2 to S1 (a3) by switching $S 1$ off and $S 2$ on. In the case of simultaneously turning on the two signals and the two pumps, it is found that data exchange between the two signals (S1 to S2: (b4), S2 to S1: (a4)) within the gated pump pulse duration is successfully realized.

We further demonstrate the single-PPLN-based channelselective data exchange for multiple WDM channels at $40 \mathrm{Gbit} / \mathrm{s}$. Four WDM channels (S1: $1535.5 \mathrm{~nm}$, S2: $1539.4 \mathrm{~nm}, \mathrm{~S} 3: 1543.3 \mathrm{~nm}, \mathrm{~S} 4: 1547.2 \mathrm{~nm}$ ) are employed in the experiment. It is possible to perform a channel-selective data exchange by simply tuning the wavelength of the two pumps. Figure 6 displays the measured typical eye diagrams and BER performance for channel-selective data exchange between WDM channels. The power penalty of $40 \mathrm{Gbit} / \mathrm{s}$ channel-selective data exchange is estimated to be less than $4 \mathrm{~dB}$ at a BER of $10^{-9}$.

Figure 7 illustrates the concept and principle for singlePPLN-based tributary channel exchange between two WDM high-speed OTDM signals $[37,38]$. A PPLN waveguide is employed as the nonlinear device to perform the tributary channel exchange. Two WDM high-speed signals (S1, S2) each consisting of many low-speed time-division multiplexed tributary channels (e.g., $1610 \mathrm{Gbit} / \mathrm{s}$ tributary channels for $160 \mathrm{Gbit} / \mathrm{s}$ signal), together with two synchronized subrate clock (e.g., $10 \mathrm{GHz}$ ) pumps, are launched into the PPLN waveguide for the tributary channel exchange. The wavelengths of two signals and two pumps are properly arranged to be symmetric ( $\mathrm{S} 1$ and $\mathrm{P} 1, \mathrm{~S} 2$ and $\mathrm{P} 2$ ) with respect to the QPM wavelength of PPLN. Inside the PPLN waveguide, two signals and two pumps participate in the cSFG/DFG nonlinear interactions, in which the photons of S1 (S2) and P1 (P2) are annihilated to produce the photons of SF wave, which are simultaneously consumed to generate the photons of S2 (S1) and P2 (P1). Due to the signal depletion and wavelength conversion effects, with the proper adjustment of pump powers, S1 can be depleted and converted to S2. Similarly, S2 can be extinguished to generate S1. As a result, data exchange between two signals (S1, S2) can be implemented. In particular, by exploiting two synchronized subrate (e.g., $10 \mathrm{GHz}$ ) clock pumps which are time aligned to one of the tributary channels of two WDM high-speed OTDM signals (e.g., $160 \mathrm{Gbit} / \mathrm{s})$, it is possible to achieve the tributary channel exchange (e.g., $10 \mathrm{Gbit} / \mathrm{s}$ ) between two WDM highspeed OTDM signals (e.g., $160 \mathrm{Gbit} / \mathrm{s}$ ). As an example shown 


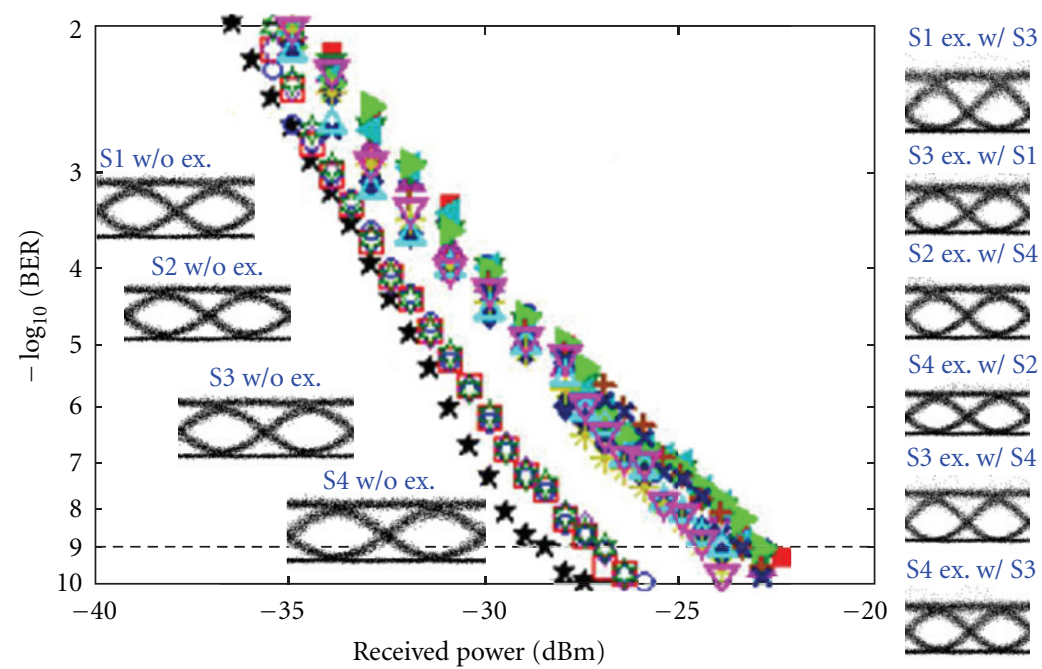

\begin{tabular}{|c|c|c|c|}
\hline B-to-B & - S2 ex. w/ S1 & $x$ & S3 ex. w/ S2 \\
\hline S1 w/o ex. & a S1 ex. w/ S3 & $\diamond$ & S2 ex. w/ S4 \\
\hline S2 w/o ex. & $\nabla \mathrm{S} 3 \mathrm{ex} . \mathrm{w} / \mathrm{S} 1$ & * & S4 ex. w/ S2 \\
\hline S3 w/o ex. & S1 ex. w/ S4 & $\triangle$ & S3 ex. w/ S4 \\
\hline S4 w/o ex. & $>\mathrm{S} 4 \mathrm{ex} . \mathrm{w} / \mathrm{S} 1$ & $\nabla$ & S4 ex. w/ S \\
\hline S1 ex. w/ S2 & + S2 ex. w/ S3 & & \\
\hline
\end{tabular}

FIGURE 6: Measured eye diagrams and BER performance of $40 \mathrm{Gbit} / \mathrm{s}$ time- and channel-selective data exchange between four WDM channels.

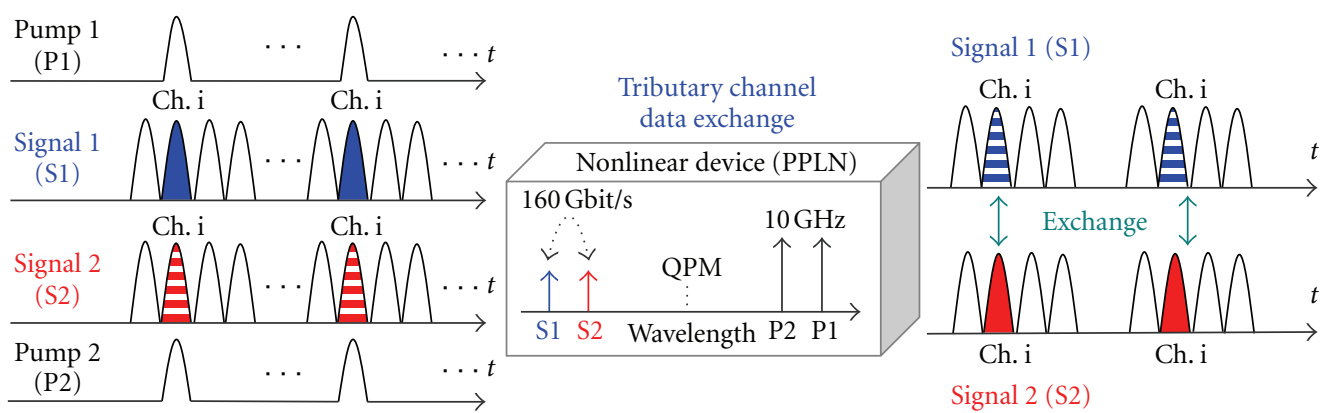

FIGURE 7: Concept and principle for single-PPLN-based tributary channel exchange between two WDM high-speed OTDM signals.

in Figure 7, the tributary channel i (Ch. i) of two WDM high-speed OTDM signals is exchanged by using the signal depletion and wavelength conversion effects of cSFG/DFG in a PPLN waveguide.

Shown in Figure 8 is the experiment setup for singlePPLN-based $10 \mathrm{Gbit} / \mathrm{s}$ tributary channel exchange between two $160 \mathrm{Gbit} / \mathrm{s}$ signals. A $500 \mathrm{~m}$ piece of HNLF with a zerodispersion wavelength (ZDW) of $\sim 1565 \mathrm{~nm}$ is employed for broadband supercontinuum generation fed with a $10 \mathrm{GHz}$ mode-locked laser (MLL) $(\sim 1551 \mathrm{~nm})$ with a pulse width of $\sim 1.9$ ps. In order to provide two $160 \mathrm{Gbit} / \mathrm{s}$ signals and two $10 \mathrm{GHz}$ pumps, the supercontinuum spectrum is divided into two branches. The first branch is sent to an amplitude modulator (AM) driven by a $10 \mathrm{Gbit} / \mathrm{s} 2^{31}-1$ pseudorandom binary sequence (PRBS) and then time-division multiplexed to $160 \mathrm{Gbit} / \mathrm{s}$ through a fiber-based polarization-maintained
10 -to-160 Gbit/s multiplexer. Two $160 \mathrm{Gbit} / \mathrm{s}$ signals at different wavelengths are further obtained simply by filtering the spectrum at 1535.6 (S1) and $1542.1 \mathrm{~nm}$ (S2) with $2 \mathrm{~nm}$ band-pass filters (BPFs). Erbium-doped fiber amplifiers (EDFAs) followed by $5 \mathrm{~nm}$ BPFs are used to boost the optical power and suppress the EDFA-induced amplified spontaneous emission (ASE) noise. The second branch is utilized for the generation of two $10 \mathrm{GHz}$ subrate clock pumps by directly slicing the supercontinuum spectrum at 1559.2 (P2) and $1565.3 \mathrm{~nm}$ (P1), respectively. Two $160 \mathrm{Gbit} / \mathrm{s}$ signals together with two $10 \mathrm{GHz}$ clock pumps are coupled into a PPLN waveguide to participate in the cascaded secondorder nonlinear interactions for the tributary channel exchange. The PPLN waveguide has a QPM wavelength of $\sim 1550.52 \mathrm{~nm}$ working at $92.1^{\circ} \mathrm{C}$. After the tributary channel exchange, the $160 \mathrm{Gbit} / \mathrm{s}$ signal is demultiplexed to $10 \mathrm{Gbit} / \mathrm{s}$ 


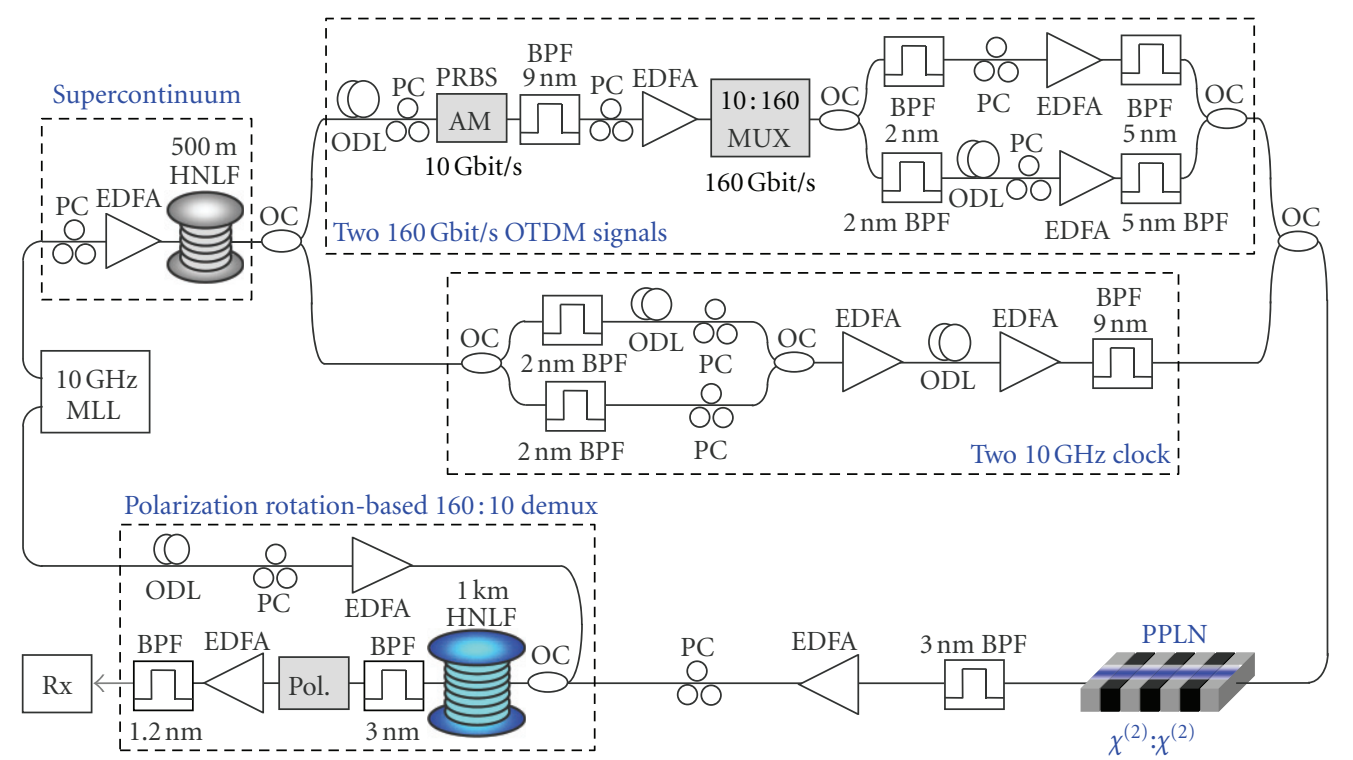

FIGURE 8: Experimental setup for single-PPLN-based $10 \mathrm{Gbit} / \mathrm{s}$ tributary channel exchange between two $160 \mathrm{Gbit} / \mathrm{s}$ signals. MLL: modelocked laser; EDFA: erbium-doped fiber amplifier; ODL: optical tunable delay line; AM: amplitude modulator; BPF: band-pass filter; PC: polarization controller; OC: optical coupler; MUX: multiplexing; Pol.: polarizer; Rx: receiver.

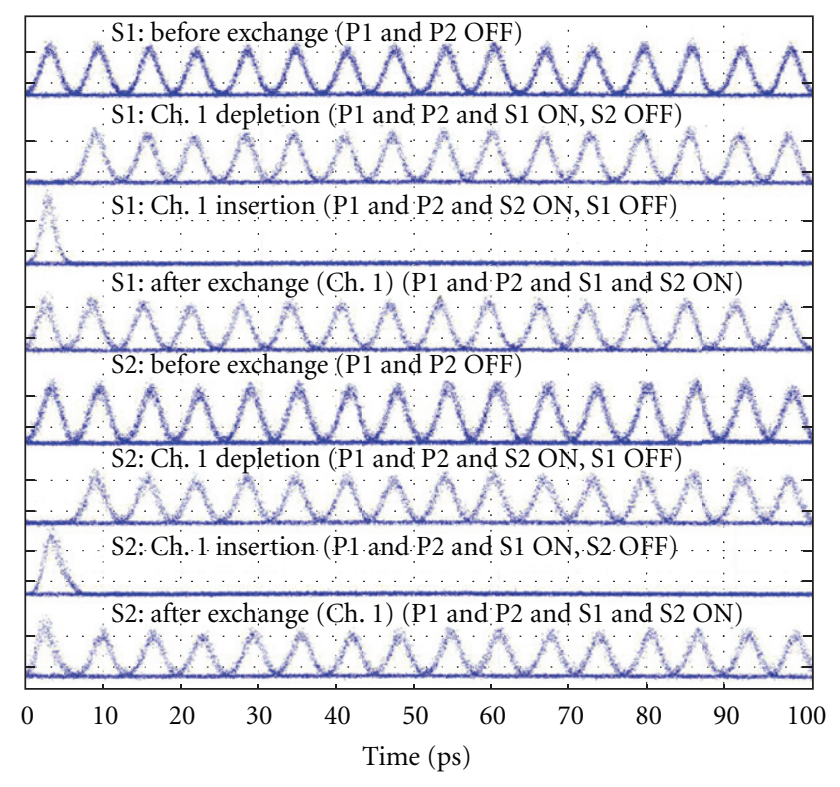

Figure 9: Measured eye diagrams for the tributary channel exchange (Ch.1).

for BER measurements by use of the Kerr effect-induced nonlinear polarization rotation in a $1-\mathrm{km}$ piece of HNLF with a ZDW of $\sim 1552 \mathrm{~nm}$.

Figure 9 displays the eye diagrams for tributary channel exchange (Ch.1) measured by an optical sampling scope. Two $10 \mathrm{GHz}$ clock pumps are time aligned to the tributary Ch.1 of two $160 \mathrm{Gbit} / \mathrm{s}$ signals. When the two pumps and S1 are present while S2 is absent, Ch.1 of S1 is depleted and converted to the Ch. 1 of S2 with the proper adjustment of pump powers and polarization states due to the signal depletion and wavelength conversion effects. Similarly, as the two pumps and S2 are turned on while S1 is off, Ch.1 of S2 is extinguished with its data information copied onto the Ch.1 of S1. The signal depletion is measured to be $\sim 17.3 \mathrm{~dB}$. In the presence of two $10 \mathrm{GHz}$ pumps and both two $160 \mathrm{Gbit} / \mathrm{s}$ signals, Ch. 1 of S2 is exchanged to the Ch. 1 of S1. Meanwhile, Ch.1 of S1 is swapped to the Ch.1 of S2, resulting in the implementation of $10 \mathrm{Gbit} / \mathrm{s}$ tributary channel exchange between two $160 \mathrm{Gbit} / \mathrm{s}$ signals. Moreover, it is convenient to further perform the $10 \mathrm{Gbit} / \mathrm{s}$ tributary exchange for all 16 tributary channels of two $160 \mathrm{Gbit} / \mathrm{s}$ signals simply by time shifting the $10 \mathrm{GHz}$ clock pumps to be aligned with the corresponding tributary channel of interest.

Figure 10 plots the BER performance for tributary channel exchange. As shown in Figures 10(a) and 10(b), compared to the back-to-back $10 \mathrm{Gbit} / \mathrm{s}$ signal, less than $3 \mathrm{~dB}$ power penalty at a BER of $10^{-9}$ is introduced for the tributary channels of the $160 \mathrm{Gbit} / \mathrm{s}$ signal before exchange. Such degradation of signal quality can be ascribed to the nonlinear effects during the supercontinuum generation and the 160to-10 Gbit/s demultiplexing. Approximately an additional $4 \mathrm{~dB}$ power penalty at a BER of $10^{-9}$ is observed for the tributary channel exchange, which is mainly caused by the beating effects between the newly converted signal and the original residual signal. Figures 10 (c) and $10(\mathrm{~d})$ further depict the power penalty of tributary exchange between two $160 \mathrm{Gbit} / \mathrm{s}$ signals for all 16 tributary channels. During the tributary channel exchange between two $160 \mathrm{Gbit} / \mathrm{s}$ signals, the average power penalty and the fluctuation of 16 tributary channels is around 3.7 and $1.1 \mathrm{~dB}$ for $\mathrm{S} 1$ (S2 to $\mathrm{S} 1$ ) and 3.9 and $1.1 \mathrm{~dB}$ for S2 (S1 to S2).

The proposed single-PPLN-based tributary channel exchange can potentially be further applied to $640 \mathrm{Gbit} / \mathrm{s}$ 

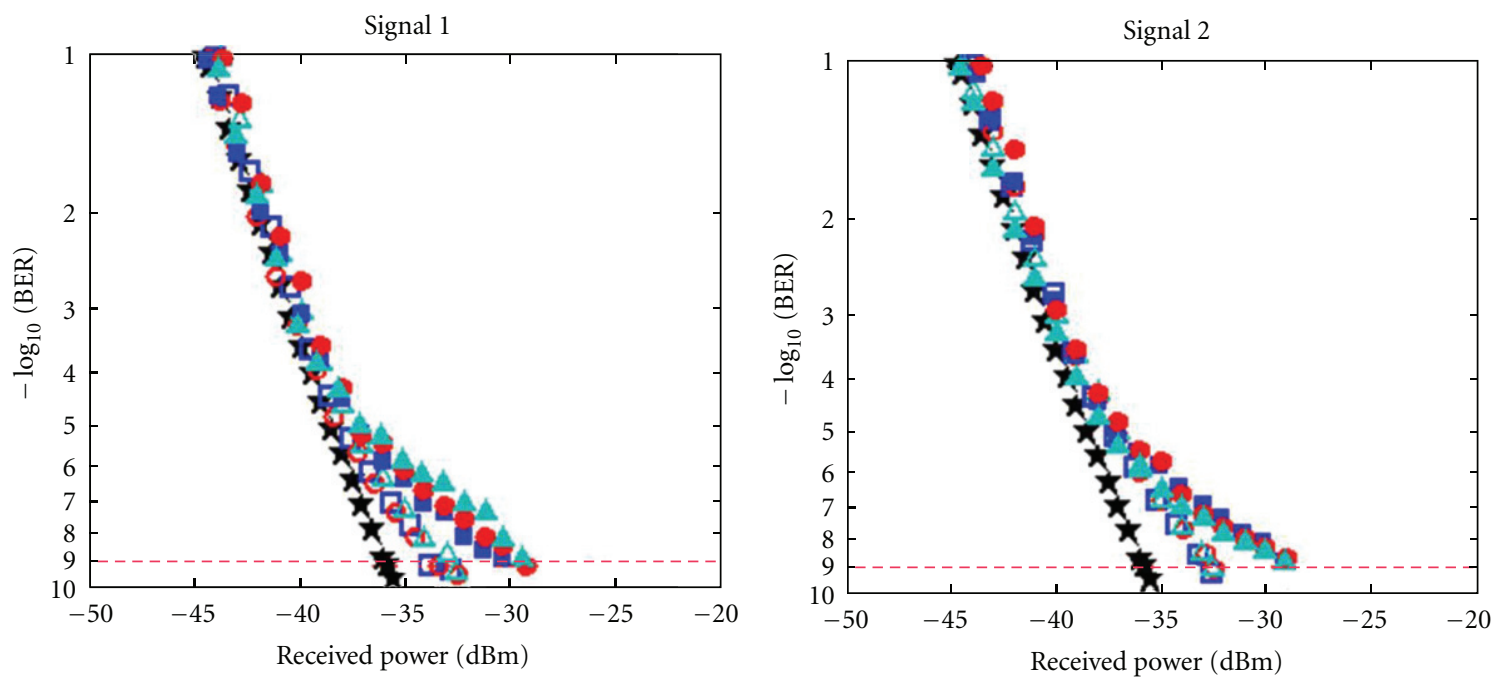

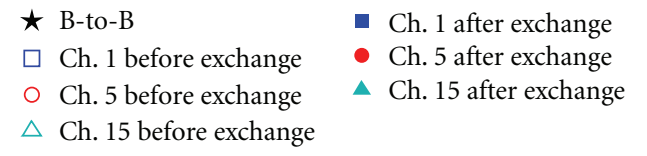

(a)

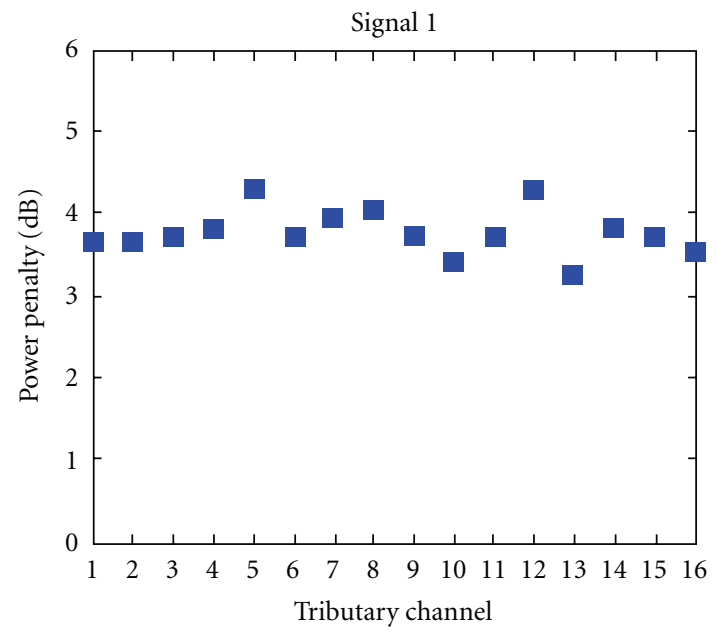

(c)

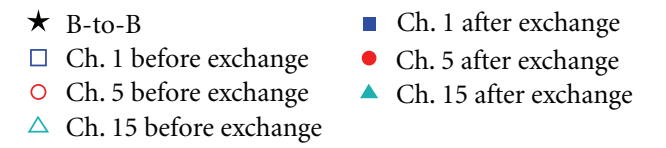

(b)

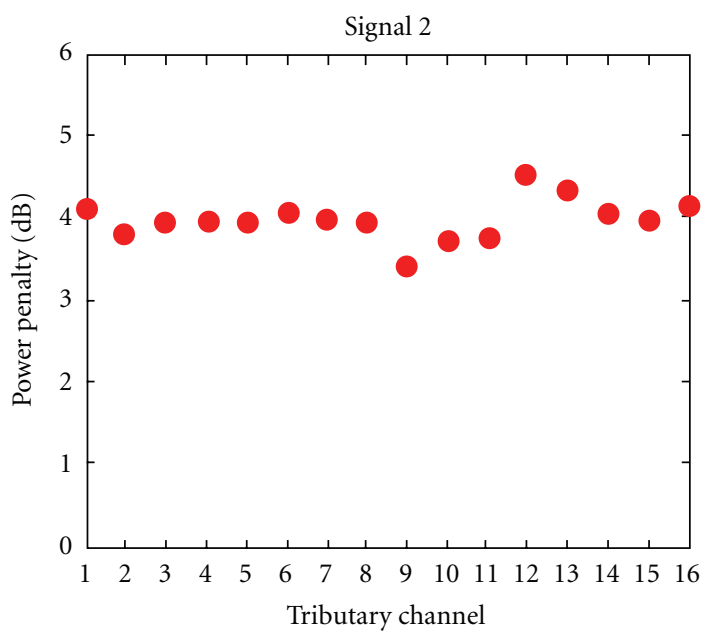

(d)

FIGURE 10: ( $\mathrm{a}$ and b) BER performance for the tributary channel exchange. (c and d) Power penalties of tributary exchange for 16 tributary channels. (a and c) Signal 1. (b and d) Signal 2.

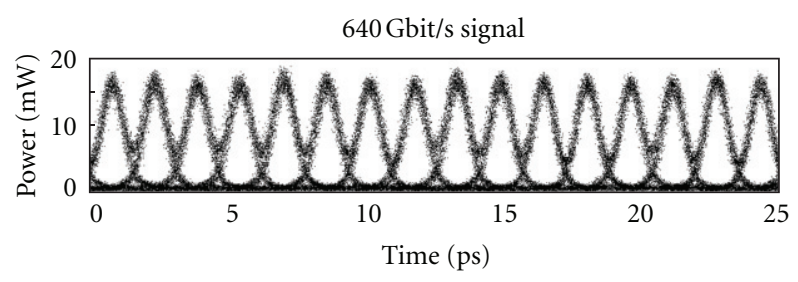

FIGURE 11: Eye diagram of the $640 \mathrm{Gbit} / \mathrm{s}$ OTDM signal.

OTDM signals. Shown in Figure 11 is the eye diagram of the $640 \mathrm{Gbit} / \mathrm{s}$ OTDM signal measured by an optical sampling scope [53]. To obtain the $640 \mathrm{Gbit} / \mathrm{s}$ OTDM signal, a
$40 \mathrm{Gbit} / \mathrm{s}$ signal is first prepared by applying data modulation to a $40 \mathrm{GHz}$ short-pulse mode-locked laser. The signal is amplified and sent to a $200 \mathrm{~m}$ piece of HNLF, with a dispersion of $\sim-0.85 \mathrm{ps} / \mathrm{nm}$ at $1550 \mathrm{~nm}$ and a dispersion slope of $\sim 0.01 \mathrm{ps} / \mathrm{nm}^{2} / \mathrm{km}$, for supercontinuum generation. A $9 \mathrm{~nm}$ band-pass filter is used for spectrum slicing to obtain a compressed copy of the $40 \mathrm{Gbit} / \mathrm{s}$ signal with a pulse width of $\sim 0.8$ ps and a root mean square (RMS) jitter of $\sim 50 \mathrm{fs}$. The $40 \mathrm{Gbit} / \mathrm{s}$ signal is then multiplexed to a $640 \mathrm{Gbit} / \mathrm{s}$ OTDM data stream using a passive optical multiplexer [53]. Remarkably, PPLN-based tributary channel exchange relies on the signal depletion and wavelength conversion effects in the cSFG/DFG nonlinear interactions, in which sum-frequency 


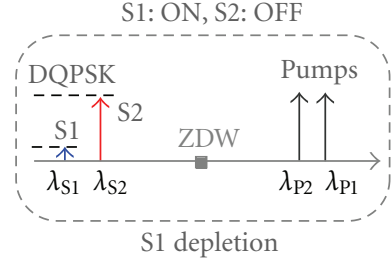

(a)

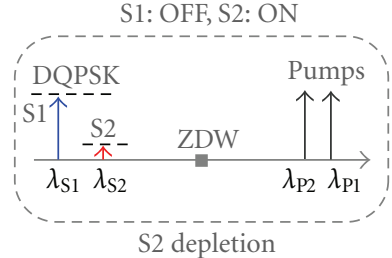

(b)

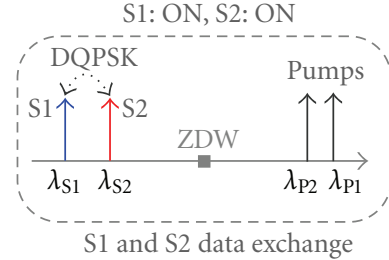

(c)

Figure 12: Concept and principle of nondegenerate FWM-based signal depletion and data exchange. (a) Signal 1 (S1) depletion. (b) Signal 2 (S2) depletion. (c) S1, S2 data exchange.

wave is also inevitably generated. Because of the temporal walkoff effect due to the group velocity mismatch (GVM) between the signals and pumps in the $1.5 \mu \mathrm{m}$ band and sumfrequency wave in the $0.77 \mu \mathrm{m}$ band, signal distortion may happen during the signal depletion and wavelength conversion processes. The longer the PPLN waveguide, the severer the temporal walk-off. Such effect could severely impact on the performance of tributary channel exchange which becomes more challengeable especially for ultra-high-speed OTDM signals (e.g., $640 \mathrm{Gbit} / \mathrm{s}$ ). With future improvement, in order to enable the PPLN-based tributary channel exchange between $640 \mathrm{Gbit} / \mathrm{s}$ signals, it is highly desirable to optimize the parameters of PPLN waveguide to alleviate the temporal walkoff effect. The expected solutions include the shortening of PPLN waveguide and the design for a quasigroup-velocity matching device in an integrated PPLN waveguide structure [54].

\subsection{Modulation-Format-Transparent Data Exchange Using} Nondegenerate FWM in an HNLF [39-42]. In addition to cSFG/DFG $\left(\chi^{(2)}: \chi^{(2)}\right)$ in a PPLN waveguide [34-38], signal depletion and wavelength conversion of nondegenerate FWM $\left(\chi^{(3)}\right)$ in an HNLF can also enable the data exchange [23-31, 39-42]. As shown in Figure 12(a), when signal 1 $\left(\mathrm{S} 1: \lambda_{\mathrm{S} 1}\right)$ and two continuous-wave (CW) pumps (P1: $\lambda_{\mathrm{P} 1}$, $\left.\mathrm{P} 2: \lambda_{\mathrm{P} 2}\right)$ are sent through the HNLF with S1 and P1 set symmetrically with respect to the ZDW of the HNLF, S1 and P1 photons are consumed to produce photons of signal 2 (S2: $\left.\lambda_{\mathrm{S} 2}\right)$ and P2 during the nondegenerate FWM process. Thus, the depletion of $\mathrm{S} 1$ is expected with its data information transparently copied onto a newly generated S2. Similarly, as shown in Figure 12(b), the depletion of S2 accompanied by the generation of S1 can be achieved as S2 and two pumps are launched into the HNLF. As shown in Figure 12(c), in the presence of two signals and two pumps at the input of HNLF with $\mathrm{S} 1(\mathrm{~S} 2)$ and $\mathrm{P} 1(\mathrm{P} 2)$ symmetric relative to the ZDW of the HNLF, S1(S2) can be extinguished and converted to S2(S1), resulting in the implementation of data exchange between $S 1$ and $S 2$.

For the nondegenerate FWM-based data exchange, pu$\mathrm{mp}$ phase modulation is adopted in the experiment to suppress the stimulated Brillouin scattering (SBS) effect so that pump power can be efficiently utilized. Previous works of nondegenerate FWM-based data exchange have been reported for OOK signals [23-31], which are not affected by the phase modulation of two pumps. In order to perform phasetransparent data exchange for DPSK and DQPSK signals, it is desired that nondegenerate FWM-based data exchange has the characteristic of modulation-format transparency.

Under the nondepletion approximation, we derive the analytical solutions for the nondegenerate FWM involving two signals and two pumps written as [40]

$$
\begin{aligned}
A_{\mathrm{SA}}^{\prime}= & \left\{A_{\mathrm{SA} 0}\left[\cos (g z)-\frac{i k \sin (g z)}{2 g}\right]\right. \\
& \left.+A_{\mathrm{SB} 0} \frac{2 i \gamma}{g} A_{\mathrm{P} 10}^{*} A_{\mathrm{P} 20} \sin (g z)\right\} e^{i\left[2 \gamma\left(\mathrm{P}_{10}+\mathrm{P}_{20}\right)+k / 2\right] z}, \\
A_{\mathrm{SB}}^{\prime}= & \left\{A_{\mathrm{SA} 0} \frac{2 i \gamma}{g} A_{\mathrm{P} 10} A_{\mathrm{P} 20}^{*} \sin (g z)+A_{\mathrm{SB} 0}\right. \\
& \left.\times\left[\cos (g z)+\frac{i k \sin (g z)}{2 g}\right]\right\} e^{i\left[2 \gamma\left(\mathrm{P}_{10}+\mathrm{P}_{20}\right)-k / 2\right] z},
\end{aligned}
$$

where $g=\sqrt{4 \gamma^{2} \mathrm{P}_{10} \mathrm{P}_{20}+k^{2} / 4}$ and $k=\Delta \beta+\gamma\left(\mathrm{P}_{10}-\mathrm{P}_{20}\right)$ are constants related to the pump powers $\left(\mathrm{P}_{10}, \mathrm{P}_{20}\right)$, nonlinear coefficient $(\gamma)$, and phase mismatching $(\Delta \beta)$. $A_{\mathrm{SA} 0}, A_{\mathrm{SB} 0}$, $A_{\mathrm{P} 10}$, and $A_{\mathrm{P} 20}$ are the complex amplitudes of input signals (SA, SB) and pumps (P1, P2) containing both amplitude and phase information. $A_{\mathrm{SA}}^{\prime}$ and $A_{\mathrm{SB}}^{\prime}$ are the complex amplitudes of output signals (SA, SB) after the data exchange. Under the exchange condition of phase matching $(k=0)$ and $g z=(N+1 / 2) \pi(N=0,1,2, \ldots)$ enabled by the proper adjustment of pump powers, we can further simplify (5a) and (5b) as follows:

$$
\begin{aligned}
& A_{\mathrm{SA}}^{\prime}= \pm A_{\mathrm{SB} 0} \frac{2 i \gamma}{g} A_{\mathrm{P} 10}^{*} A_{\mathrm{P} 20} e^{i 2 \gamma\left(\mathrm{P}_{10}+\mathrm{P}_{20}\right) z} \\
& A_{\mathrm{SB}}^{\prime}= \pm A_{\mathrm{SA} 0} \frac{2 i \gamma}{g} A_{\mathrm{P} 10} A_{\mathrm{P} 20}^{*} e^{i 2 \gamma\left(\mathrm{P}_{10}+\mathrm{P}_{20}\right) z} .
\end{aligned}
$$

Note that (6a) and (6b) indicate the linear relationship of complex amplitude between the output and input signals $\left(A_{\mathrm{SA}}^{\prime} \propto A_{\mathrm{SB} 0}, A_{\mathrm{SB}}^{\prime} \propto A_{\mathrm{SA} 0}\right)$, implying the implementation of phase-transparent optical data exchange. We can further obtain the corresponding phase relationships of $\varphi_{\mathrm{SA}}{ }^{\prime}=\varphi_{\mathrm{SB}}+$ $\varphi_{\mathrm{P} 2}-\varphi_{\mathrm{P} 1}$ and $\varphi_{\mathrm{SB}}{ }^{\prime}=\varphi_{\mathrm{SA}}+\varphi_{\mathrm{P} 1}-\varphi_{\mathrm{P} 2}$. Remarkably, the pump 


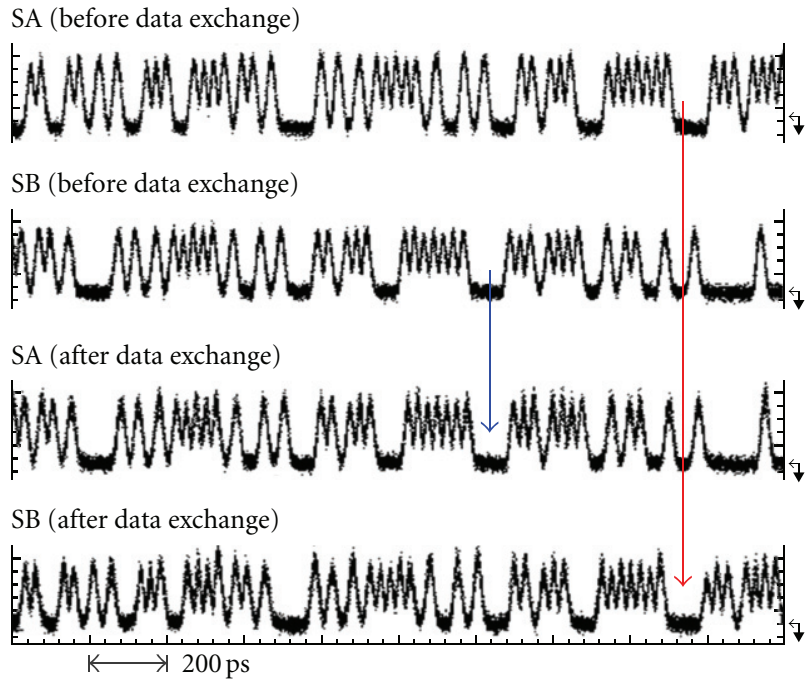

FIGURE 13: Measured destructively demodulated AMI waveforms for data exchange between two $40 \mathrm{Gbit} / \mathrm{s}$ NRZ-DPSK signals.

phase transfer $\left(\varphi_{\mathrm{P} 1}-\varphi_{\mathrm{P} 2} \neq 0\right)$ to the exchanged signals does not impact the OOK data exchange but could cause severe degradation on the DPSK/DQPSK data exchange. Fortunately, according to the deduced phase relationships, it is possible to cancel the pump phase transfer by applying the precisely identical phase modulation to the two pumps (i.e., $\left.\varphi_{\mathrm{P} 1}=\varphi_{\mathrm{P} 2}\right)$, which makes it applicable to implement the data exchange of DPSK/DQPSK signals.

A $1-\mathrm{km}$ piece of HNLF is adopted in the experiment, which has a nonlinear coefficient of $9.1 \mathrm{~W}^{-1} \cdot \mathrm{km}^{-1}$, a ZDW of $\sim 1552 \mathrm{~nm}$, and a fiber loss of $0.45 \mathrm{~dB} / \mathrm{km}$. To suppress SBS, identical phase modulation is applied to the two pumps using a single phase modulator (PM) driven by a $10 \mathrm{Gbit} / \mathrm{s}$ PRBS. According to (6a) and (6b), the precisely identical phase modulation of the two pumps could be canceled in the output signals after data exchange.

We first demonstrate the phase-transparent data exchange between two $40 \mathrm{Gbit} / \mathrm{s} 2^{31}$-1 PRBS NRZ-DPSK signals (SA: signal A, SB: signal B) $[39,40]$. In order to verify the NRZ-DPSK data exchange, Figure 13 displays the destructively demodulated alternate-mark inversion (AMI) waveforms. It can be clearly seen that the data information of SA and SB is swapped, and therefore phase-transparent data exchange between two $40 \mathrm{Gbit} / \mathrm{s}$ NRZ-DPSK signals is successfully implemented. Shown in Figure 14 is the BER performance of $40 \mathrm{Gbit} / \mathrm{s}$ NRZ-DPSK data exchange. The power penalty of wavelength conversion with only SA or SB on is less than $1.5 \mathrm{~dB}$. An additional $3 \mathrm{~dB}$ power penalty is introduced when combining together the newly converted signal and the residual signal with both SA and SB on. Thus, an approximate $4.5 \mathrm{~dB}$ total power penalty at a BER of $10^{-9}$ is obtained for $40 \mathrm{Gbit} / \mathrm{s} \mathrm{NRZ-DPSK} \mathrm{data} \mathrm{exchange.}$

We further demonstrate the phase-transparent data exchange between two $100 \mathrm{Gbit} / \mathrm{s} 2^{7}$-1 PRBS RZ-DQPSK signals (S1: signal 1, S2: signal 2) [41, 42]. Figure 15 displays the measured temporal waveforms of the demodulated inphase (Ch. I) and quadrature (Ch. Q) components for the
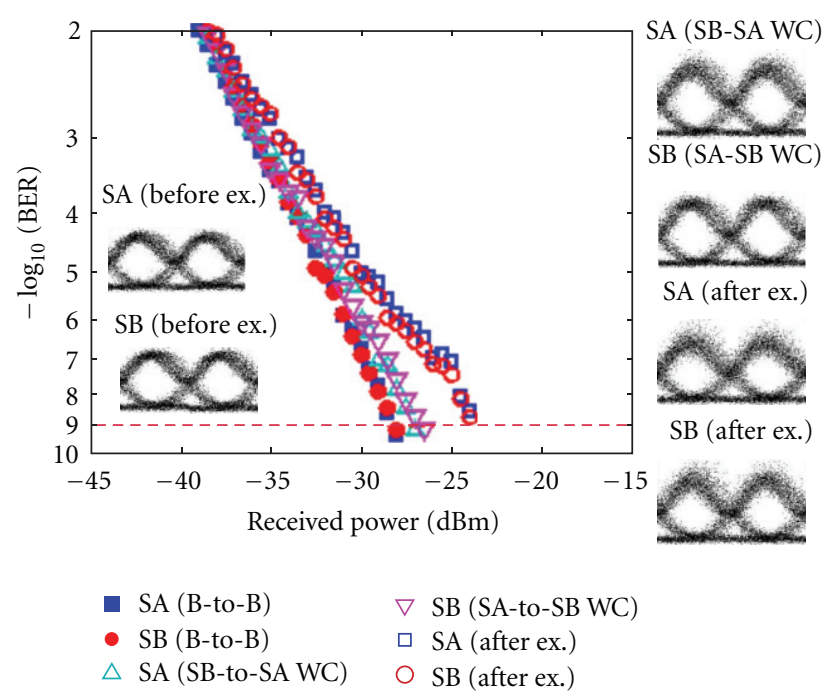

FIGURE 14: Measured eyes and BER performance of data exchange between two $40 \mathrm{Gbit} / \mathrm{s}$ NRZ-DPSK signals.

$100 \mathrm{Gbit} / \mathrm{s}$ DQPSK data exchange. It can be clearly observed that the data information carried by two $100 \mathrm{Gbit} / \mathrm{s}$ RZDQPSK signals is successfully swapped after the nondegenerate FWM-based data exchange. In addition, by comparing the waveforms after wavelength conversion (i.e., only S1 or S2 is present) and after data exchange (i.e., both $\mathrm{S} 1$ and S2 are present), we can observe the degradation of waveforms after data exchange with added noise, which can be ascribed to the beating effect of in-band interference between the newly converted signal and the original residual signal. Figure 16 shows the measured constellation diagrams of different signals for the $100 \mathrm{Gbit} / \mathrm{s}$ DQPSK data exchange. Four phase levels of all $100 \mathrm{Gbit} / \mathrm{s}$ RZ-DQPSK signals are observed.

Figure 17 plots the BER curves for the $100 \mathrm{Gbit} / \mathrm{s}$ DQPSK data exchange. Less than $1.2 \mathrm{~dB}$ power penalty at a BER of $10^{-9}$ is obtained for the $100 \mathrm{Gbit} / \mathrm{s}$ DQPSK wavelength conversion with only one signal (S1 or S2) present. Less than $5 \mathrm{~dB}$ power penalty at a BER of $10^{-9}$ is observed for the $100 \mathrm{Gbit} / \mathrm{s}$ DQPSK data exchange. The extra power penalty of data exchange compared to wavelength conversion could be ascribed to the beating effect between the newly converted signal and the original residual signal.

We investigate the tolerance of the temporal pump phase misalignment and the dynamic range of the input signal power for the $100 \mathrm{Gbit} / \mathrm{s}$ RZ-DQPSK data exchange. Figure 18 depicts the impact of time misalignment between two phase-modulated pumps on the performance of wavelength conversion and data exchange (i.e., relative power penalty compared to the case of perfect pump phase alignment). It is found that the performance of the wavelength conversion and data exchange suffers rapid degradation as the pump phase misalignment goes beyond \pm 2 ps. Such phenomena can be briefly explained as follows. Large temporal pump phase misalignment causes incomplete pump phase cancellation, and thus the resultant residual pump phase transfer to the phase noise degrades the performance of the wavelength conversion and data exchange. 


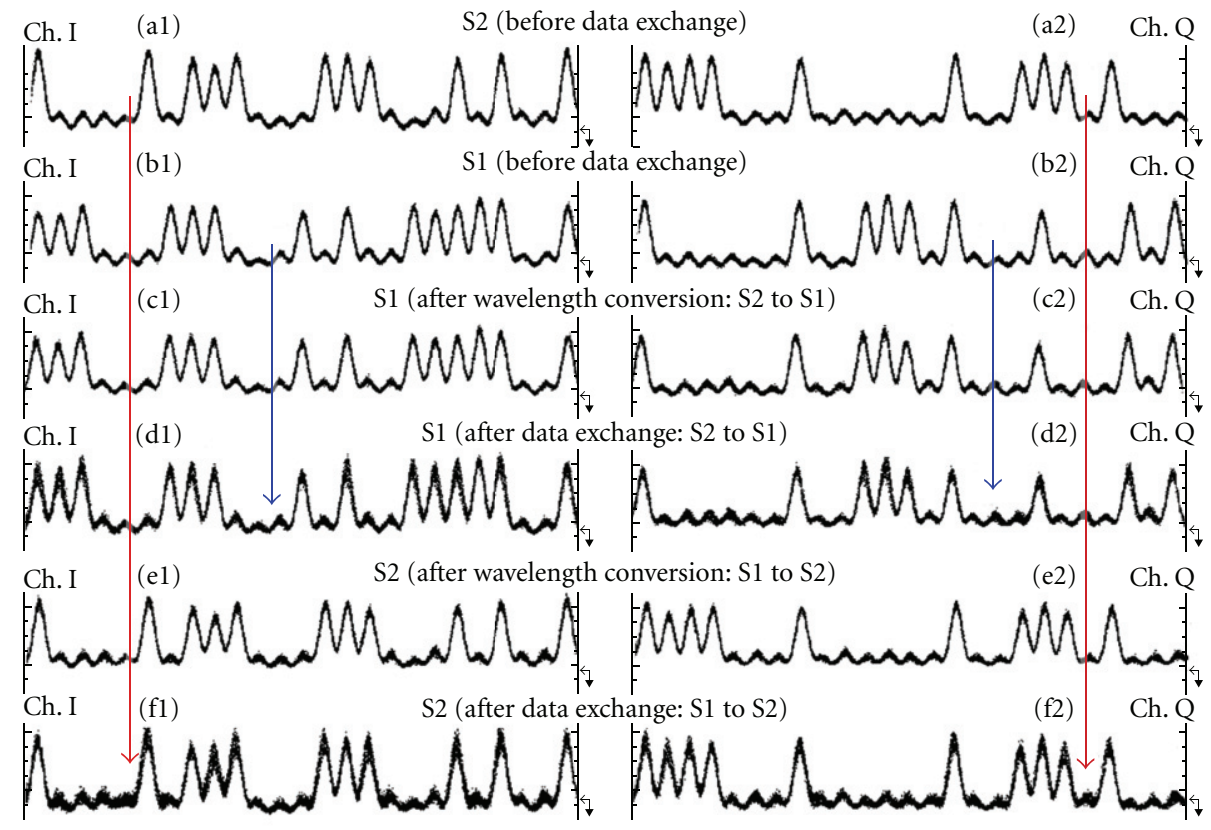

FIGURe 15: Demodulated waveforms (Ch. I and Ch. Q) for $100 \mathrm{Gbit} / \mathrm{s}$ DQPSK data exchange. (a1 and a2) S1 before exchange (P1: OFF, P2: OFF). (b1 and b2) S2 before exchange (P1: OFF, P2: OFF). (c1 and c2) S1 after wavelength conversion (WC: S2 to S1) (S1: OFF, S2: ON, P1: ON, P2: ON). (d1 and d2) S1 after exchange (Ex.: S2 to S1) (S1: ON, S2: ON, P1: ON, P2: ON). (e1 and e2) S2 after wavelength conversion (WC: S1 to S2) (S1: ON, S2: OFF, P1: ON, P2: ON). (f1 and f2) S2 after exchange (Ex.: S1 to S2) (S1: ON, S2: ON, P1: ON, P2: ON). (a1)-(f1) Ch. I. (a2)-(f2) Ch. Q.

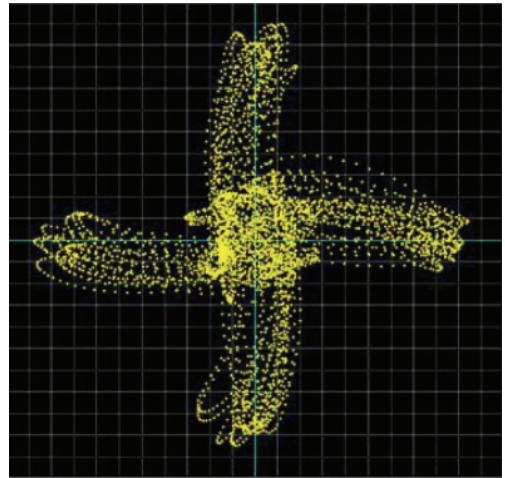

(a)

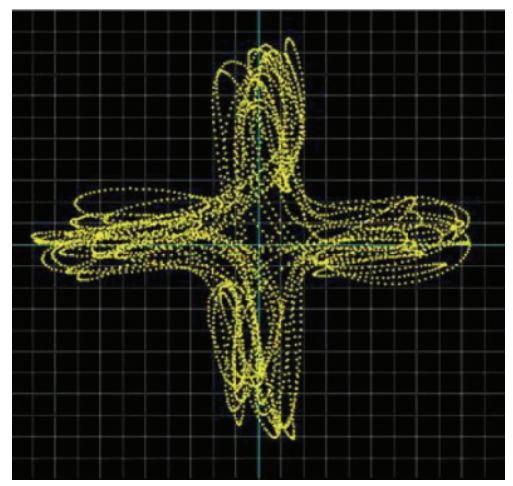

(d)

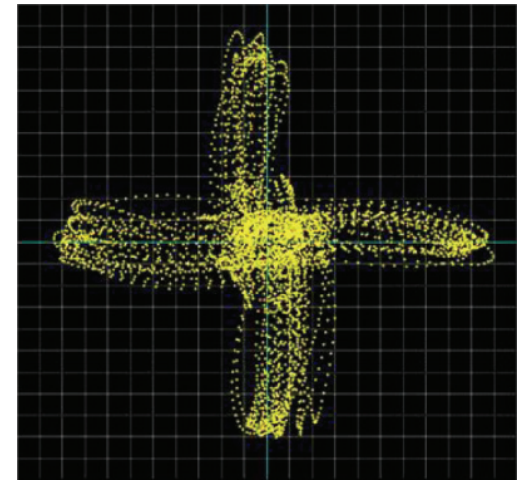

(b)

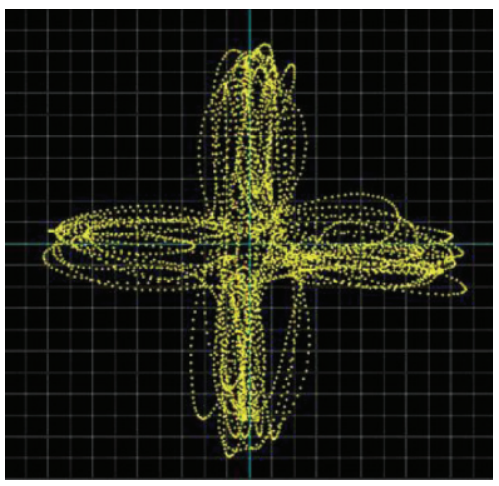

(e)

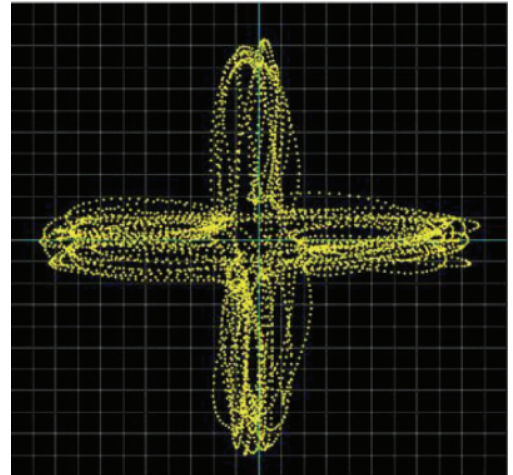

(c)

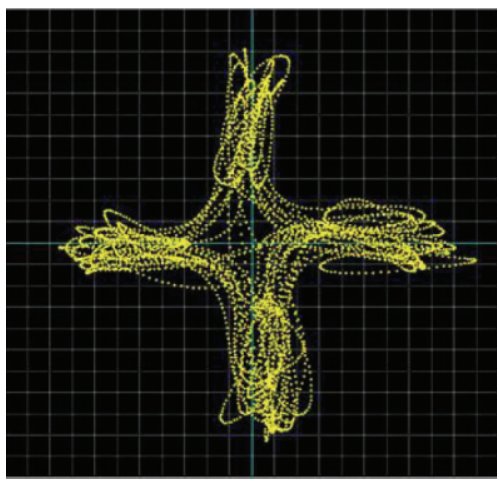

(f)

FIGURe 16: Constellation diagrams for $100 \mathrm{Gbit} / \mathrm{s}$ DQPSK data exchange. (a) S1: back-to-back. (b) S2: back-to-back. (c) S1 after wavelength conversion (WC: S2 to S1) (S1: OFF, S2: ON). (d) S2 after wavelength conversion (WC: S1 to S2) (S1: ON, S2: OFF). (e) S1 after data exchange (Ex.: S2 to S1) (S1: ON, S2: ON). (f) S2 after data exchange (Ex.: S1 to S2) (S1: ON, S2: ON). 


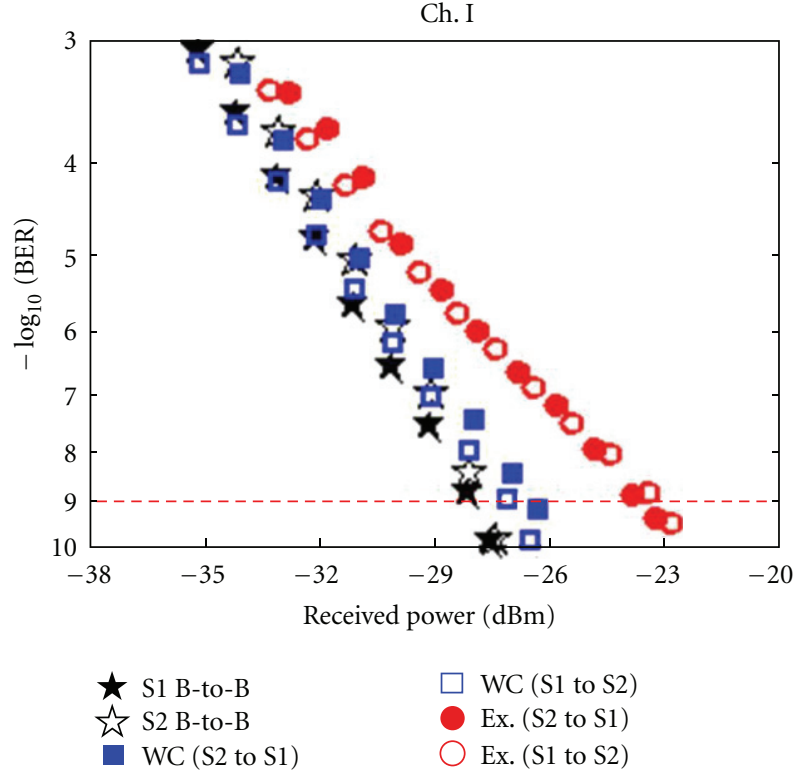

(a)
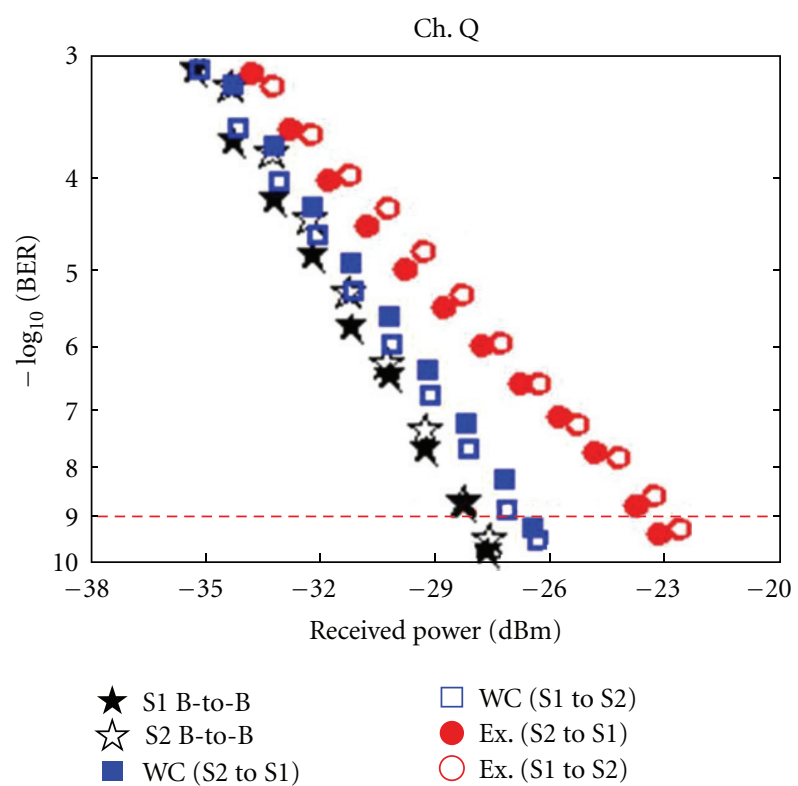

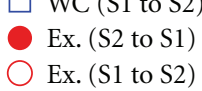

b)

FIGURE 17: BER curves for the $100 \mathrm{Gbit} / \mathrm{s}$ DQPSK data exchange.

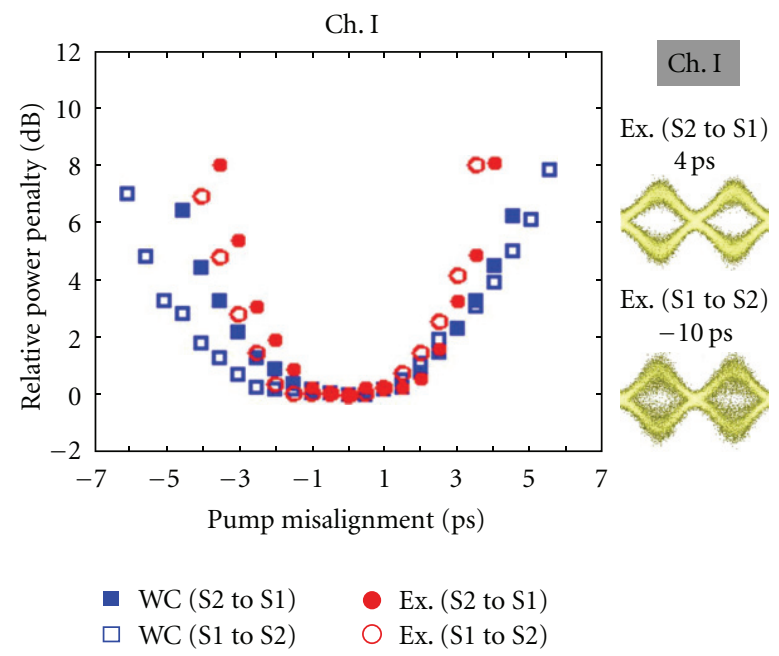

(a)

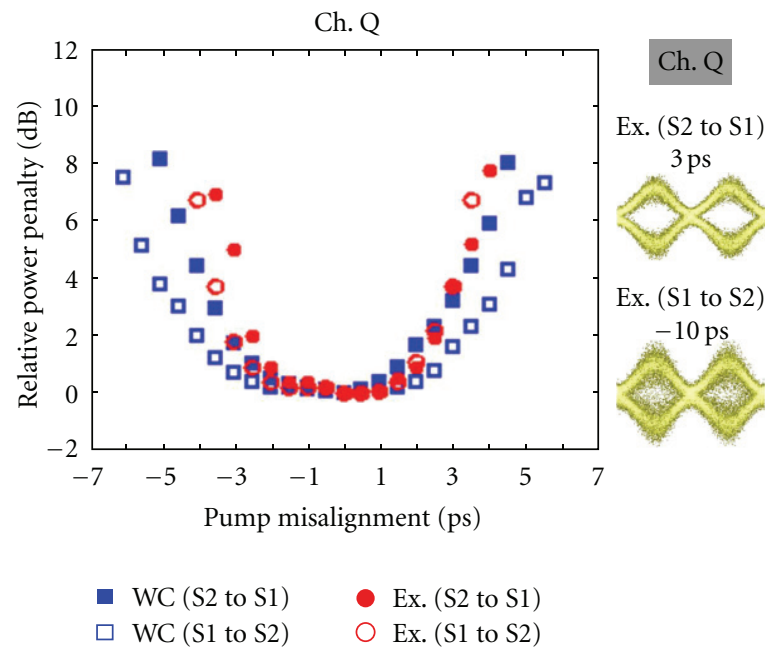

(b)

FIGURE 18: Impact of pump phase misalignment on the exchange performance for $100 \mathrm{Gbit} / \mathrm{s}$ DQPSK data exchange.

Figure 18 also displays typical balanced eye diagrams of the exchanged signals under different values of temporal pump phase misalignment. Under a larger pump phase misalignment of $-10 \mathrm{ps}$, we can see nearly complete eye closure of the exchanged signals. As a consequence, for the phase-transparent optical data exchange of $100 \mathrm{Gbit} / \mathrm{s}$ DQPSK signals, the optimal pump phase cancellation in the exchanged signals is of great importance to achieve the optimized exchange performance.

Figure 19 shows the received power at a BER of $10^{-9}$ as a function of the input signal power. As the input signal power changes from -12.0 to $8.1 \mathrm{dBm}$, the variation of the received power at a BER of $10^{-9}$ is estimated to be less than $3.5 \mathrm{~dB}$. An approximate $20 \mathrm{~dB}$ dynamic range of the input signal power is achieved for the nondegenerated FWM-based optical data exchange of $100 \mathrm{Gbit} / \mathrm{s}$ RZ-DQPSK signals.

As a side consideration, phase modulation is applied to the two pumps to suppress the SBS effect in the nondegenerate FWM-based modulation-format-transparent data exchange. Although precisely identical pump phase modulation will not affect the exchanged signals, the exchange performance becomes degraded with the increase of pump phase misalignment. With further improvement, without involving pump phase modulation, some alternative approaches could be considered to suppress the SBS effect [55], including the use of an isolator between two fiber segments [56], 


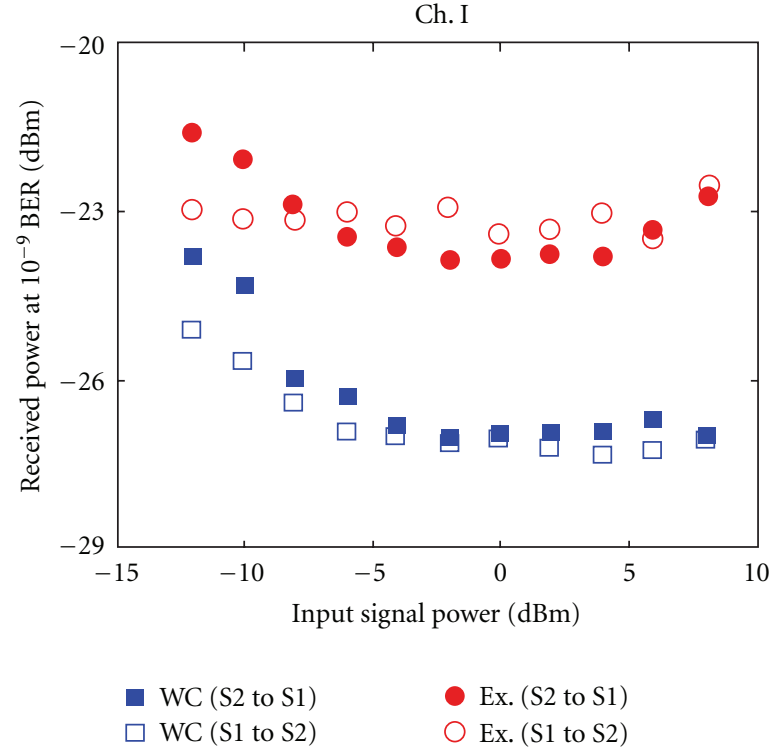

(a)

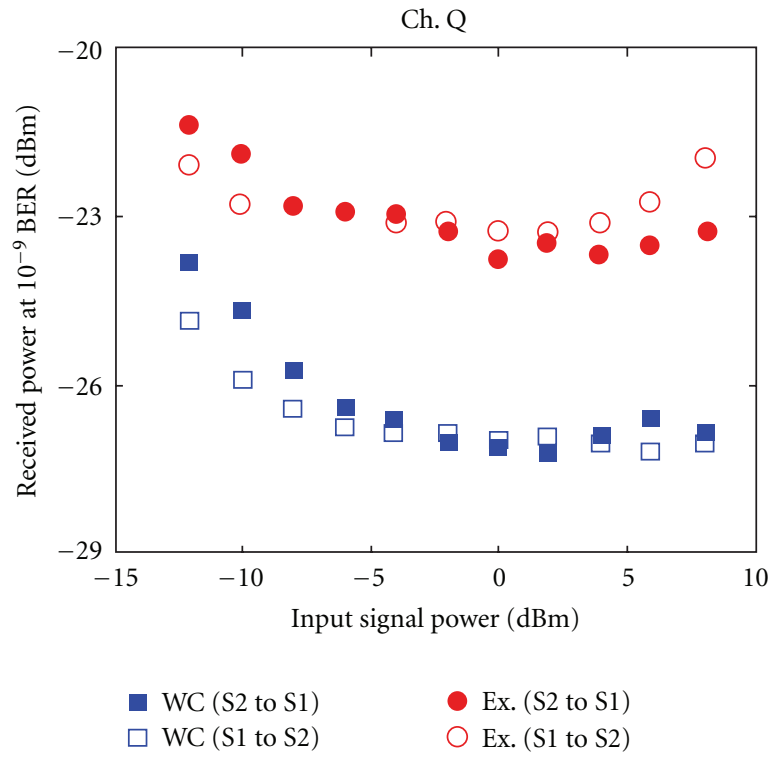

(b)

FIGURE 19: Dynamic range of the input signal power for $100 \mathrm{Gbit} / \mathrm{s}$ DQPSK data exchange.
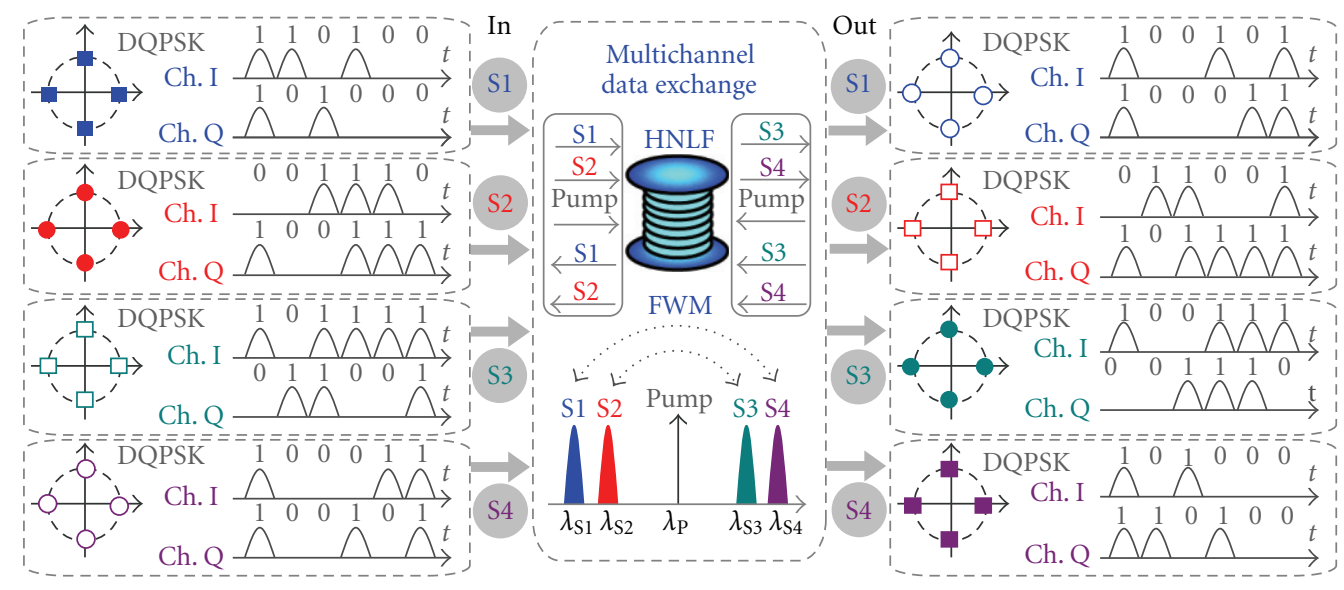

FIGURE 20: Concept and principle of simultaneous multichannel DQPSK data exchange.

strain distribution [57], temperature gradient [58, 59], particular profile design [60-62], and bismuth-oxide-based nonlinear fiber (Bi-NLF) [63]. It is expected that these alternative methods of SBS suppression could enable the use of CW pumps to achieve nondegenerate FWM-based modulation-format-transparent data exchange.

\subsection{Multichannel Data Exchange Using Bidirectional Degen-} erate FWM in an HNLF [43-46]. The aforementioned signal depletion and wavelength conversion-based schemes with two pumps enable the two-channel data exchange [34-42]. However, the extended applications to simultaneous multichannel data exchange might be limited. A laudable goal would be to explore the data exchange between multichannel signals.

Figure 20 illustrates the concept and principle of multichannel data exchange $[43,44]$. Degenerate FWM with a sin- gle CW pump is utilized. Four-channel DQPSK signals (S1-S4) are symmetric with respect to the CW pump. Simultaneous data exchange between S1 and S4 as well as S2 and S3 is expected. In general, such exchange function is not applicable with the unidirectional degenerate FWM in a single HNLF since the newly converted signals cannot be separated from the original signals. A potential solution is to explore the bidirectional degenerate FWM in a single HNLF assisted by optical filtering. As shown in Figure 20, for the input four-channel signals (S1-S4), the filtered S1, S2 and CW pump are sent to HNLF from the left side, yielding S4 and S3 via degenerate FWM. The newly generated S4 and S3 are selected at the right side of HNLF while the original S1, S2 and CW pump are blocked. Meanwhile, the filtered S3, S4 and CW pump are fed into HNLF from the right side, producing S2 and S1 by degenerate FWM. The newly converted S2 and S1 are selected at the left side of HNLF 


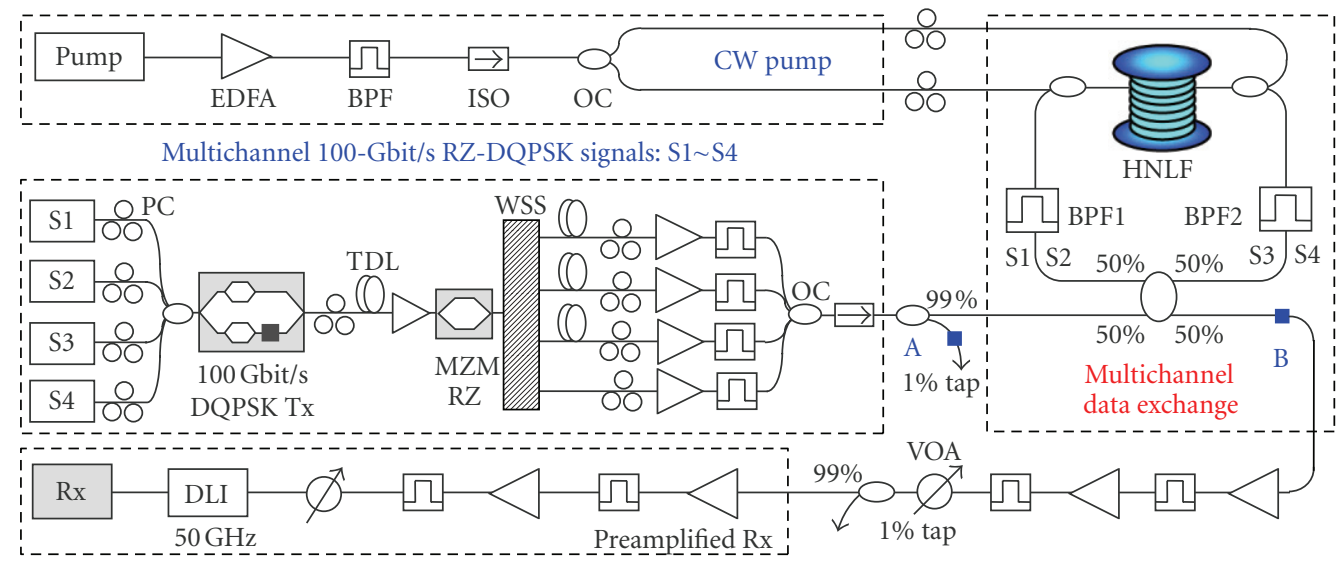

FIGURE 21: Experimental setup for multichannel DQPSK data exchange. HNLF: highly nonlinear fiber; BPF: band-pass filter; PC: polarization controller; EDFA: erbium-doped fiber amplifier; ISO: isolator; OC: optical coupler; TDL: tunable delay line; MZM: MachZehnder modulator; WSS: wavelength-selective switch; VOA: variable optical attenuator; DLI: delay line interferometer; Tx: transmitter; Rx: receiver.

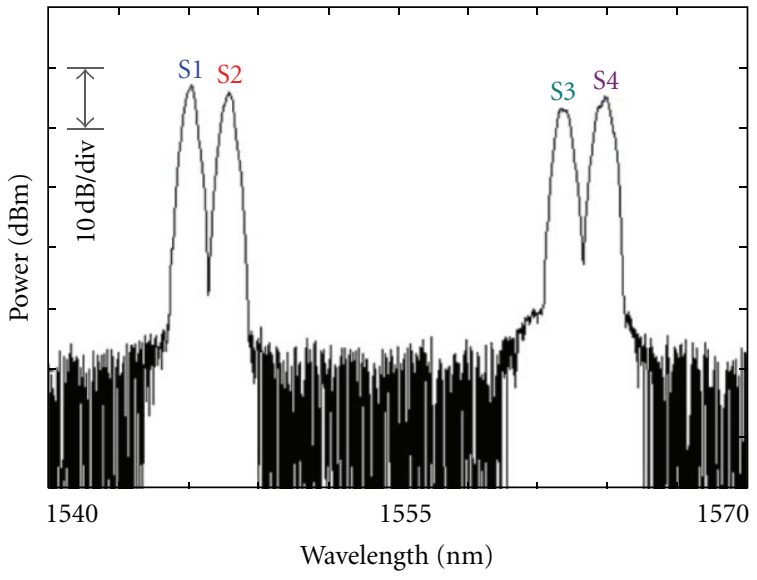

(a)

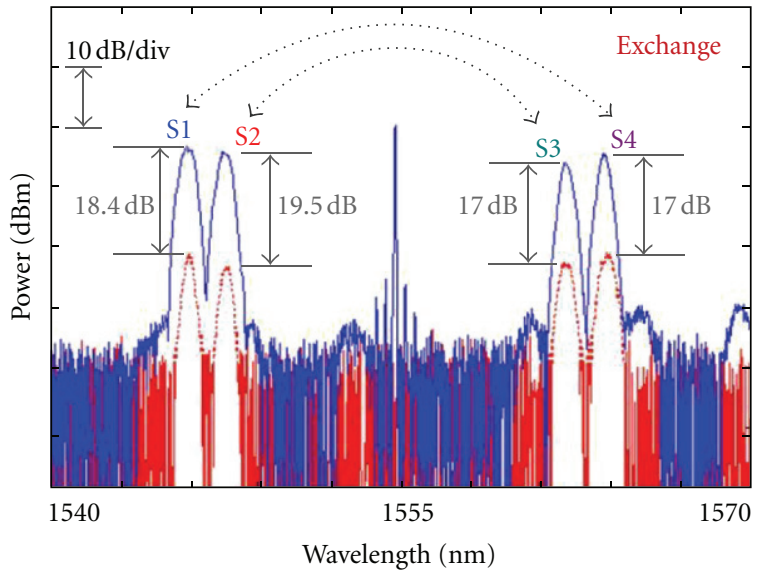

(b)

FIGURE 22: Spectra for four-channel DQPSK data exchange. (a) Input four-channel $100 \mathrm{Gbit} / \mathrm{s}$ RZ-DQPSK signals. (b) Output spectra in the absence (dashed curve: Rayleigh scattering)/presence (solid curve: after data exchange) of CW pump.

while the original S3, S4 and CW pump are removed. As a consequence, simultaneous four-channel data exchange (S1 and S4, S2 and S3) can be achieved using bidirectional FWM in a single HNLF assisted by optical filtering. The combined S1-S4 from both sides of HNLF are the output four-channel signals after data exchange. Note that the in-phase (Ch. I) and quadrature (Ch. Q) components of DQPSK signals are swapped after data exchange due to the phase-conjugation characteristic of degenerate FWM.

Figure 21 shows the experimental setup for multichannel data exchange. Four-channel $100 \mathrm{Gbit} / \mathrm{s} \quad 2^{7}-1$ PRBS RZDQPSK signals (S1-S4) are obtained by sending four tunable CW lasers to a $100 \mathrm{Gbit} / \mathrm{s}$ (50-Gsymbol/s) DQPSK transmitter (Tx) followed by a RZ pulse carver. Four-channel RZ-DQPSK signals are then separated, relatively delayed by integral symbols, recombined, and sent to a fiber loop mirror incorporating a 460-m piece of HNLF, two band-pass filters (BPF1, BPF2), and optical couplers (OCs). The HNLF has a nonlinear coefficient of $20 \mathrm{~W}^{-1} \cdot \mathrm{km}^{-1}$, a ZDW of $\sim 1556 \mathrm{~nm}$, and a dispersion slope of $\sim 0.026 \mathrm{ps} / \mathrm{nm}^{2} / \mathrm{km}$. A CW pump is coupled into the fiber loop mirror from both sides of the HNLF to enable bidirectional degenerate FWM in a single HNLF. Four-channel signals are arranged symmetrically relative to the CW pump. Note that BPF1 (BPF2) passes S1 and S2 (S3 and S4) while blocks S3, S4 and pump (S1, S2 and pump), resulting in simultaneous multichannel data exchange between S1 and S4 as well as S2 and S3 via bidirectional degenerate FWM. At the output of the fiber loop mirror, the collected four-channel signals after data exchange are sent to a preamplified receiver $(\mathrm{Rx})$ for BER measurements. A $50 \mathrm{GHz}$ delay line interferometer (DLI) is used to demodulate the in-phase and quadrature components of $100 \mathrm{Gbit} / \mathrm{s}$ DQPSK signals.

ITU-grid-compatible four-channel $100 \mathrm{Gbit} / \mathrm{s}$ RZDQPSK signals (S1: $1546.12 \mathrm{~nm}, \quad$ S2: $1547.72 \mathrm{~nm}, \quad$ S3: $1562.23 \mathrm{~nm}, \mathrm{~S} 4: 1563.86 \mathrm{~nm}$ ) are employed in the experiment for multichannel data exchange. Figure 22(a) depicts the spectrum of input four-channel $100 \mathrm{Gbit} / \mathrm{s}$ RZ-DQPSK sign- 

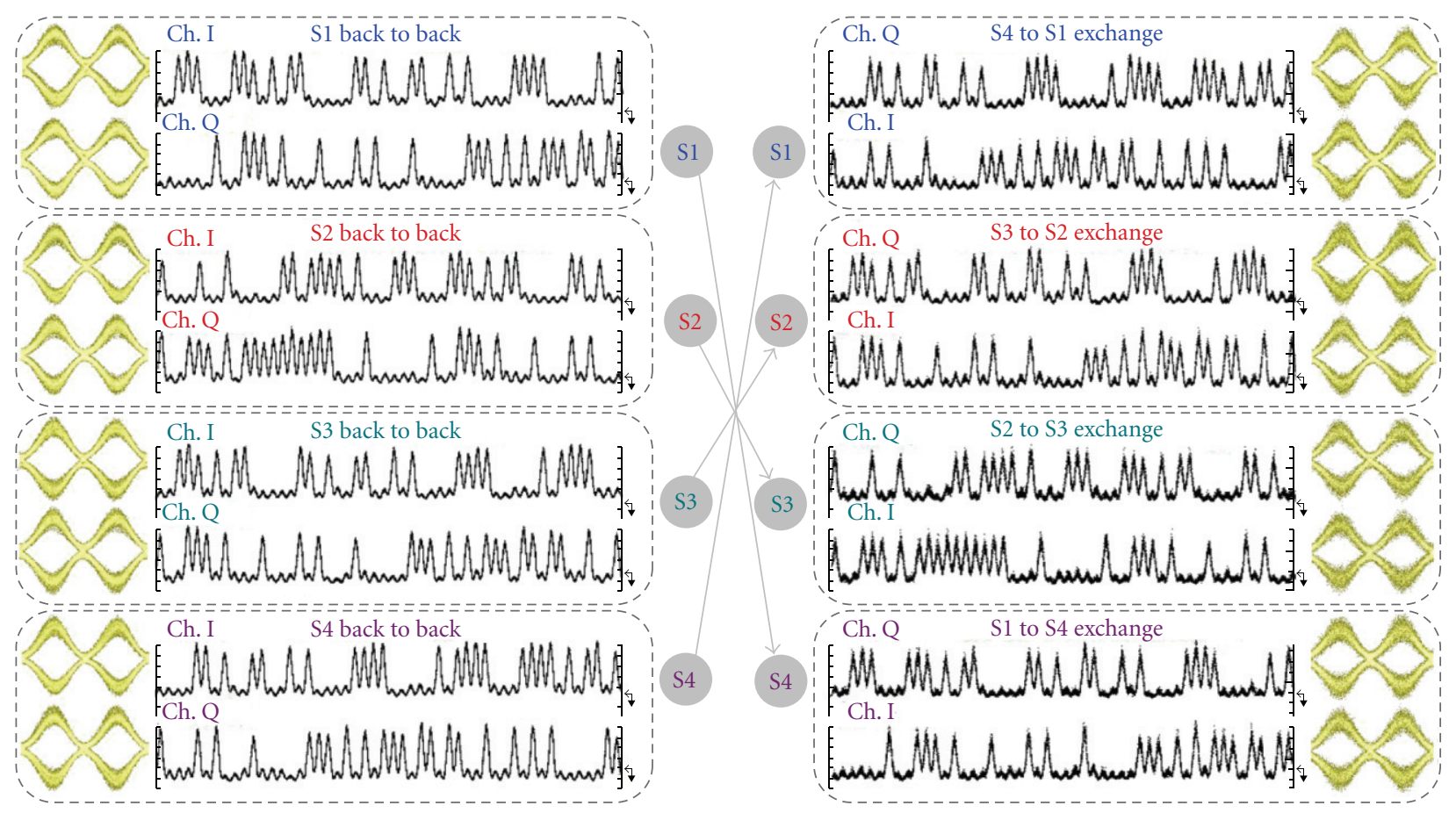

FIGURE 23: Waveforms and balanced eyes of demodulated in-phase (Ch. I) and quadrature (Ch. Q) components for four-channel $100 \mathrm{Gbit} / \mathrm{s}$ DQPSK data exchange.

als. S1(S2) and S4(S3) are symmetric with respect to the CW pump. Figure 22(b) shows the spectrum after four-channel data exchange in the presence of CW pump (solid curve). The spectrum of residual signals (i.e., resulting from Rayleigh scattering in HNLF [64]) in the absence of CW pump (dashed curve) is also depicted in Figure 22(b). Figure 23 displays temporal waveforms and balanced eyes of demodulated in-phase (Ch. I) and quadrature (Ch. Q) components of $100 \mathrm{Gbit} / \mathrm{s}$ RZ-DQPSK signals before and after data exchange. It is verified that four-channel $100 \mathrm{Gbit} / \mathrm{s} \mathrm{RZ-}$ DQPSK data exchange (S1 and S4, S2 and S3) is successfully implemented. Also, it is noted that Ch. I and Ch. Q of DQPSK signals are swapped after data exchange as a result of the phase-conjugated degenerate FWM. Figure 24 plots the BER curves for four-channel $100 \mathrm{Gbit} / \mathrm{s}$ RZ-DQPSK data exchange. Less than $4.7 \mathrm{~dB}$ power penalty is observed at a BER of $10^{-9}$, which could be caused by the beating effect between the newly exchanged signals and the original residual signals.

The proposed simultaneous multichannel data exchange can be incorporated in a reconfigurable network switching element to enhance the efficiency and flexibility of networks. We construct a reconfigurable Tbit/s network switching element using double-pass LCoS technology accompanied by bidirectional degenerate FWM in a single HNLF. We demonstrate the LCoS+HNLF-based 2.3-Tbit/s multifunctional grooming switch which performs simultaneous selective add/drop, switchable data exchange, and power equalization, for 23-channel $100 \mathrm{Gbit} / \mathrm{s}$ RZ-DQPSK signals [45, 46].

Figure 25 depicts the concept and principle of multifunctional grooming switch, which relies on the doublepass LCoS technology and bidirectional degenerate FWM inside a single HNLF. For input unequalized multichannel WDM signals, the available signal manipulations through the multifunctional grooming switch unit include (i) local selective dropping of one or more channels and adding of the corresponding channels carrying new data information, (ii) switchable data exchange between multiple channels of interest, and (iii) power equalization across all the WDM channels. An example of 7-channel WDM signals is presented in Figure 25. The core part of the setup is a wavelength selective switch (WSS) based on a two-dimensional (2D) array of LCoS pixels $[65,66]$. The phase retardance of each pixel is set by adjusting the voltages applied to the LCoS. The 2D LCoS array can be described with two axes, that is, horizontal wavelength axis and vertical displacement axis. Unequalized 7-channel $100 \mathrm{Gbit} / \mathrm{s}$ DQPSK signals are sent from an input/output fiber array (port A) to a diffraction grating, which angularly disperses each wavelength channel to a different portion of the LCoS along the horizontal wavelength axis. Vertically, the light diverges to overlap a large number of pixels (typically about 400). Independent attenuation control of optical power and spatial switch of individual wavelength channels to the desired fiber array ports (S1 to port B, S4/S5 to port $\mathrm{C}, \mathrm{S} 2 / \mathrm{S} 3$ to port $\mathrm{D}, \mathrm{S} 6 / \mathrm{S} 7$ to port $\mathrm{E}$ ) are achieved by manipulating the phase front of the $2 \mathrm{D}$ array of LCoS pixels along the vertical axis. Multiple grooming functions on the channels of interest are then applied to different fiber array ports. As shown in Figure 25, in addition to the power equalization of all channels enabled by attenuation control, wavelength add/drop at port B and data exchange between port $\mathrm{D}$ and port $\mathrm{E}$ are adopted. In order to perform simultaneous multichannel data exchange between S2 and S7 


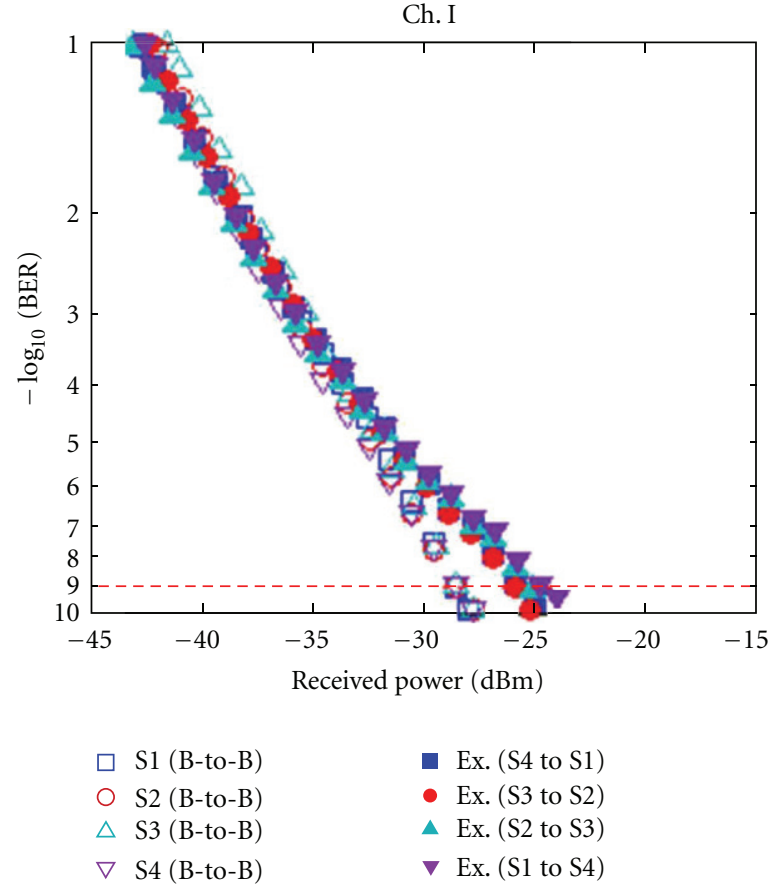

(a)

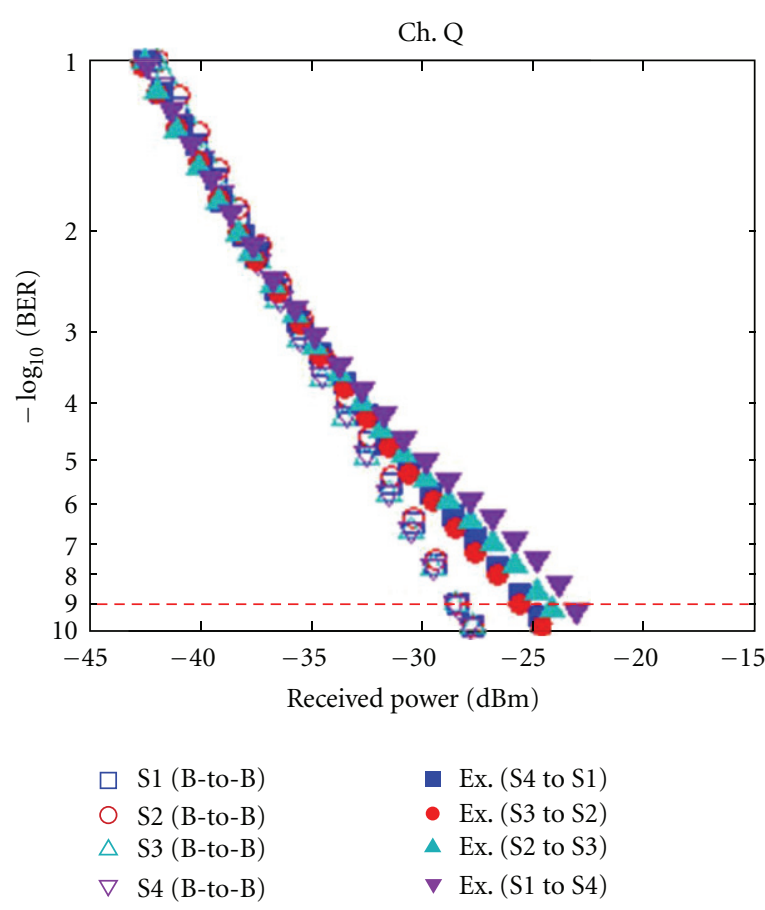

(b)

FIGURE 24: BER curves for simultaneous four-channel $100 \mathrm{Gbit} / \mathrm{s}$ DQPSK data exchange.

as well as S3 and S6, bidirectional degenerate FWM in a single HNLF is employed. After data exchange, the information carried by different channels is swapped. In order to deliver the newly added channels and exchanged channels together, the LCoS device is utilized in a double-pass fashion such that fiber array port A not only delivers input unequalized signals but also exports output signals after grooming switch. In addition to the channels undergoing add/drop (S1) and data exchange (S2, S3, S6, S7), other channels (S4, S5) are also kept and sent back by a fiber loop mirror through port $\mathrm{C}$. The dropped channel (S1) is achieved at port B. Optical circulators are adopted to assist the double-pass operation. Considering the dashed boxes in Figure 25 as an LCoS+HNLF-based switch unit, reconfigurable multifunctional grooming switch (simultaneous add/drop, data exchange, and power equalization) is enabled by the double-pass programmable LCoS and bidirectional nonlinear interactions in a single HNLF.

ITU-grid-compatible 23-channel (from S1: $1531.12 \mathrm{~nm}$ to S23: $1566.31 \mathrm{~nm}) 100 \mathrm{Gbit} / \mathrm{s}$ RZ-DQPSK signals are employed in the experiment. Figure 26 shows the measured spectrum of the input unequalized 23-channel $100 \mathrm{Gbit} / \mathrm{s}$ RZ-DQPSK signals with a power fluctuation of $\sim 9.1 \mathrm{~dB}$. Shown in the insets are typical balanced eyes for the in-phase (Ch. I) and quadrature (Ch. Q) components.

In addition to single-channel add/drop and switchable two-channel data exchange $[45,46]$, we also demonstrate 2.3-Tbit/s grooming switch with two-channel add/drop and six-channel data exchange. Shown in Figure 27 is the measured spectrum and typical balanced eyes after grooming switch with power equalization $(<1 \mathrm{~dB})$ for all 23 channels (input unequalization: $\sim 9.1 \mathrm{~dB}$ ), two-channel add/drop for

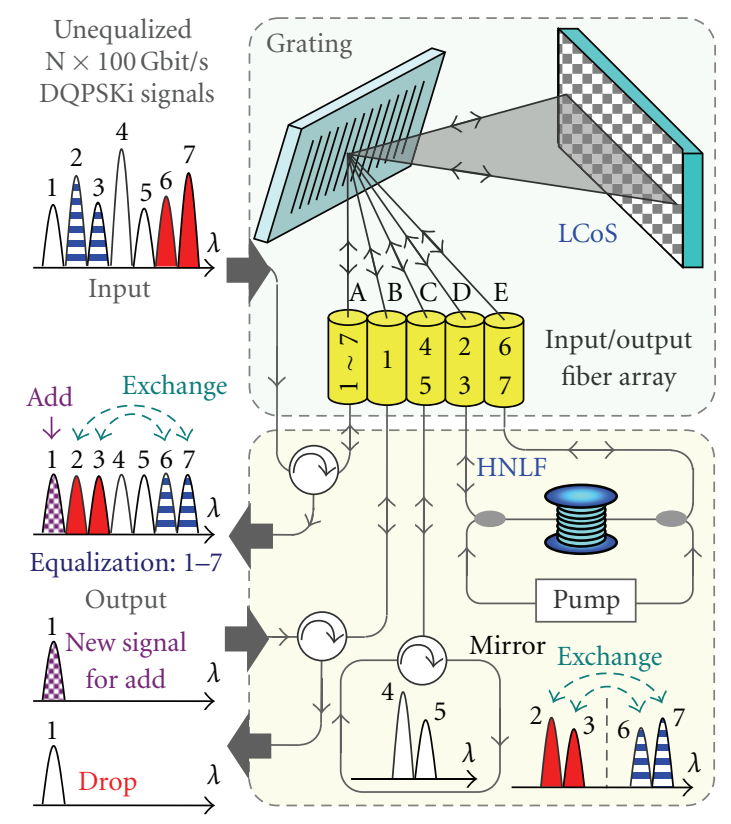

FIGURE 25: Concept and principle of LCoS+HNLF-based multifunctional grooming switch (add/drop, data exchange, power equalization).

S6 and S7, and simultaneous six-channel data exchange (S10, S11, S12, S21, S22, S23). The inset of Figure 27 depicts the spectrum of dropped S6 and S7. The BER performance is plotted in Figure 28, and power penalties less than $1.2 \mathrm{~dB}$ for two-channel add, $0.5 \mathrm{~dB}$ for two-channel drop, and $5 \mathrm{~dB}$ for 


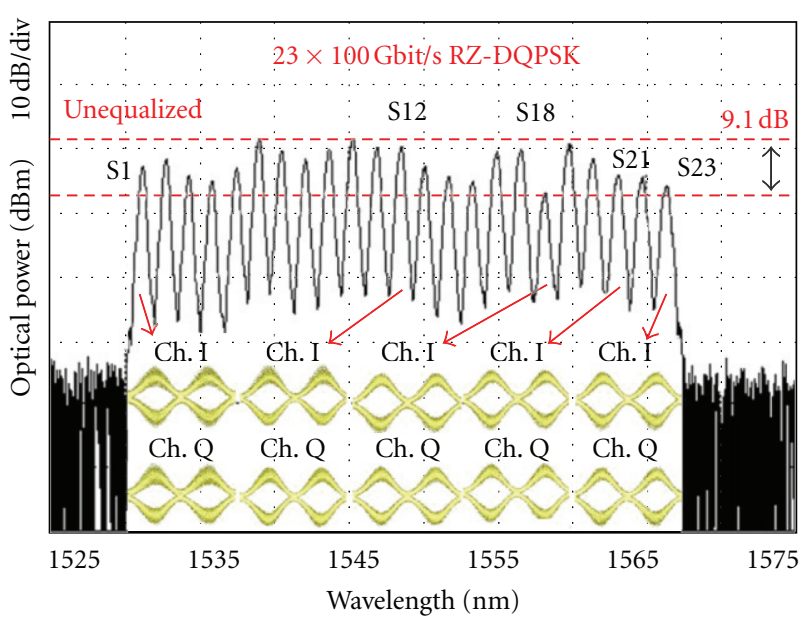

Figure 26: Measured spectrum and balanced eyes for input unequalized 23-channel $100 \mathrm{Gbit} / \mathrm{s}$ RZ-DQPSK signals.

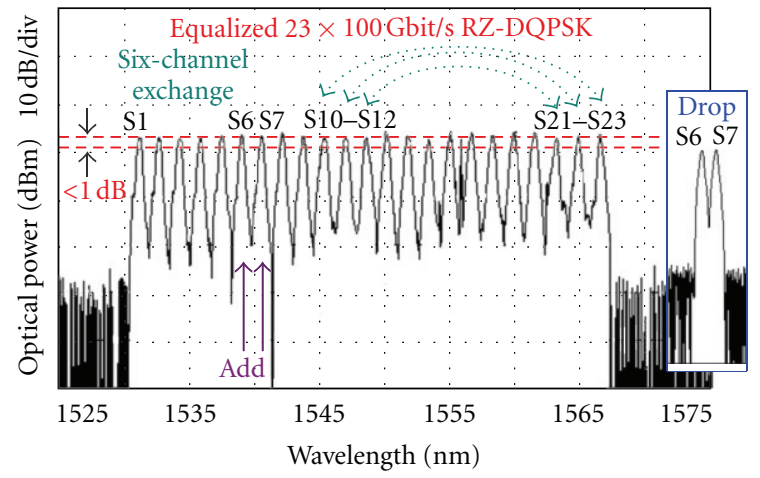

(a)

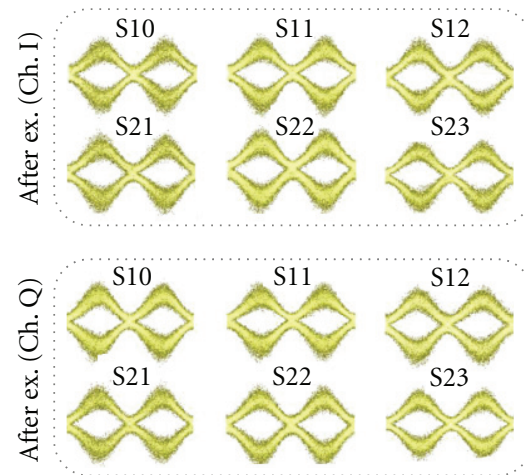

(b)

FIGURE 27: Measured spectrum and balanced eyes after multifunctional grooming switch (S6, S7: add/drop; S10, S11, S12, S21, S22, S23: data exchange; S1-S23: power equalization).

six-channel data exchange are observed at a BER of $10^{-9}$. The relatively large penalties of data exchange are mainly caused by the beating effects of in-band interference between the newly converted signal and the original residual signal.

3.4. Data Exchange between Two Orthogonal Polarizations Using Kerr-Induced Nonlinear Polarization Rotation in an HNLF $[47,48]$. In addition to the data exchange in the wavelength and time domains [34-46], it is also possible to perform data exchange between two orthogonal polarizations in the time and polarization domains [47, 48, 67]. We experimentally demonstrate the orthogonal tributary channel exchange between two pol-muxed DPSK OTDM data streams by using the Kerr effect-induced nonlinear birefringence in an HNLF $[47,48]$.

Figure 29(a) illustrates the concept and principle of DPSK OTDM signal demultiplexing (demux) based on the optical Kerr effect [20]. The DPSK OTDM signal and a strong subrate clock pump are linearly polarized at the HNLF input with a $45^{\circ}$ angle between their directions of polarization. When the pump is absent, all the tributary channels of the DPSK OTDM signal will be blocked by a crossed polarizer at the HNLF output. In the presence of the subrate clock pump, which is time aligned with one of the tributary channels of the DPSK OTDM signal, the pump-induced nonlinear birefringence via Kerr effect will cause a polarization change of the selected tributary channel. A $90^{\circ}$ polarization rotation could be available through proper adjustment of the pump power, thereby resulting in the selected tributary channel passing through the polarizer and enabling the DPSK OTDM signal demultiplexing $[68,69]$.

Figure 29(b) further depicts the schematic diagram and operation principle of the Kerr effect-based orthogonal tributary channel exchange of a pol-muxed DPSK OTDM signal. The strong subrate clock pump is $45^{\circ}$ linearly polarized with respect to the two orthogonal polarizations of a pol-muxed DPSK OTDM signal. With the help of proper pump power control, the pump-induced nonlinear birefringence by Kerr effect could bring the selected tributary channel (aligned with the subrate clock pump) to a $90^{\circ}$ polarization rotation for both of the two orthogonal polarizations of the polmuxed signal, leading to the orthogonal tributary channel exchange when the pump is present. Other unselected orthogonal tributary channels with the pump absent will not experience the nonlinear polarization rotation and hence will be untouched. In addition, simply by shifting the subrate clock pump to be aligned with the tributary channel of interest, it is possible to implement orthogonal tributary channel exchange for all tributary channels of the pol-muxed DPSK OTDM signal.

Figure 30 shows the experimental setup for the demultiplexing of a DPSK OTDM signal and the orthogonal tributary channel exchange of a pol-muxed DPSK OTDM signal. A $10 \mathrm{GHz}$ MLL at $1551.0 \mathrm{~nm}$ with a pulse width of $\sim 2.4 \mathrm{ps}$ is used to generate an $80 \mathrm{Gbit} / \mathrm{s}$ DPSK OTDM signal through a PM driven by a $10 \mathrm{Gbit} / \mathrm{s} 2^{31}-1$ PRBS and a subsequent $10: 80$ multiplexer. A $160 \mathrm{Gbit} / \mathrm{s}$ pol-muxed DPSK OTDM signal is then achieved by dividing and recombining the $80 \mathrm{Gbit} / \mathrm{s}$ DPSK OTDM signal. The polarization controllers (PCs), tunable optical delay lines (ODLs), and variable optical attenuators (VOAs) are employed to provide the orthogonal polarization control, introduce the relative time delay, and equalize the power level for a pol-muxed signal. Another $10 \mathrm{GHz}$ MLL at $1559.7 \mathrm{~nm}$ with a pulse width of $\sim 1.9$ ps serves as the subrate clock pump, which is synchronized with the $10 \mathrm{GHz}$ MLL at $1551.0 \mathrm{~nm}$. A $1-\mathrm{nm}$ BPF is used after the $10 \mathrm{GHz}$ MLL to broaden the pump pulse width (4.4 ps) 

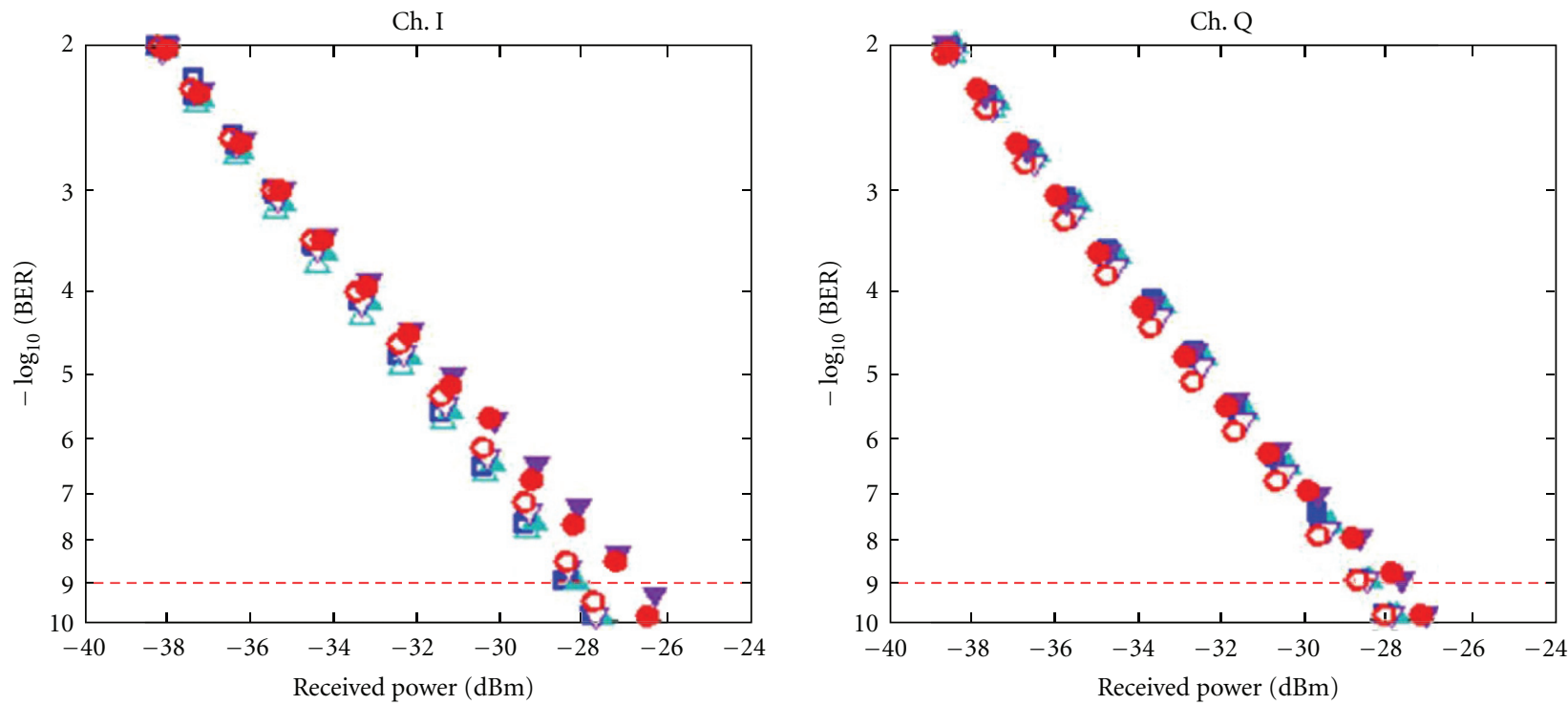

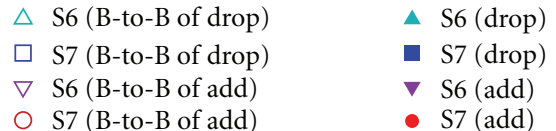

(a1)

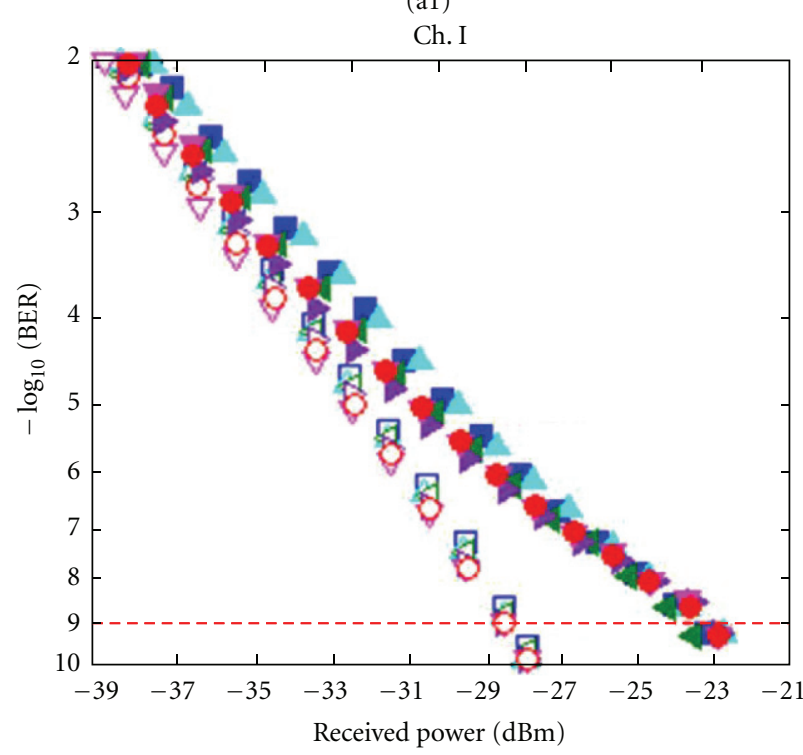

$\square$ S10 (B-to-B)

$\triangle$ S11 (B-to-B)

$\triangleleft$ S12 (B-to-B)

$\triangle$ S21 (B-to-B)

$\nabla$ S22 (B-to-B)

O S23 (B-to-B)
- Ex. (S23 to S10)

$\triangle$ Ex. (S22 to S11)

4 Ex. (S21 to S12)

- Ex. (S12 to S21)

$\checkmark$ Ex. (S11 to S22)

- Ex. (S10 to S23)

(b1)
S6 (drop)

- S7 (drop)

$\checkmark$ S6 (add)

- S7 (add)

O S7 (B-to-B of add)

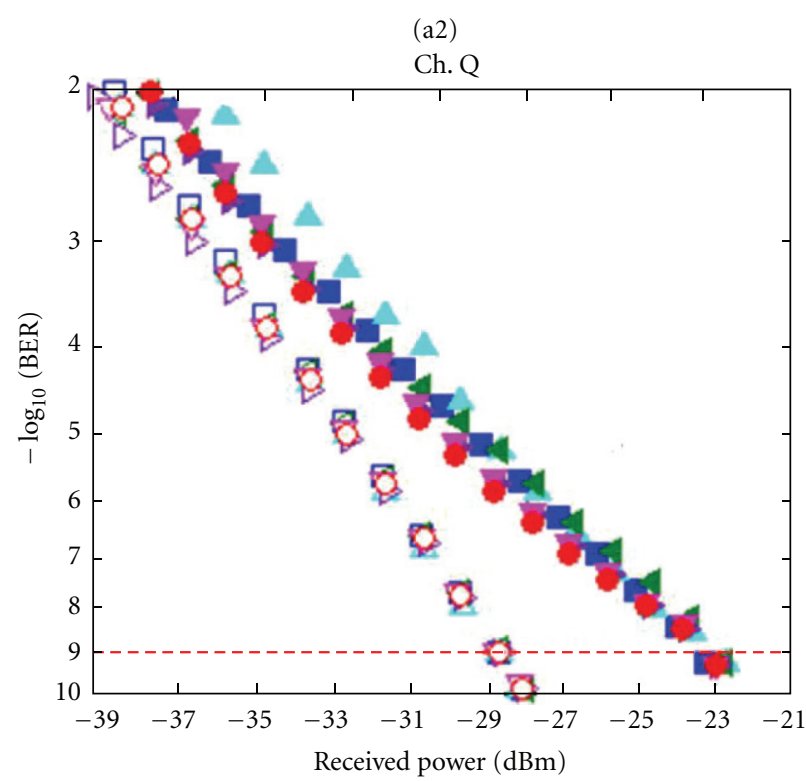

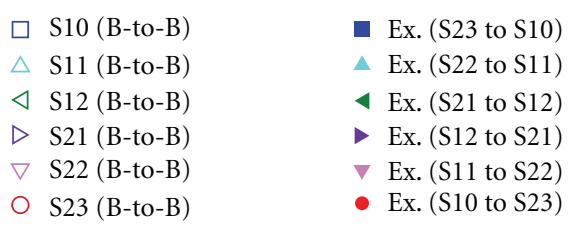

(b2)

FIGURE 28: BER curves for (a1 and a2) two-channel add/drop (S6, S7) and (b1 and b2) simultaneous six-channel data exchange (S10, S11, S12, S21, S22, S23).

for performance optimization. A 330-m piece of HNLF with a nonlinear coefficient $(\gamma)$ of $25 \mathrm{~W}^{-1} \cdot \mathrm{km}^{-1}$ and a ZDW of $\sim 1562.2 \mathrm{~nm}$ is adopted for the DPSK OTDM signal demultiplexing or the orthogonal tributary channel exchange of a pol-muxed DPSK OTDM signal. For the tributary exchange of pol-muxed DPSK OTDM, after the orthogonal tributary channel exchange, another $1-\mathrm{km}$ piece of HNLF with a $\gamma$ of $9.1 \mathrm{~W}^{-1} \cdot \mathrm{km}^{-1}$ and a ZDW of $1552 \mathrm{~nm}$ is utilized for 


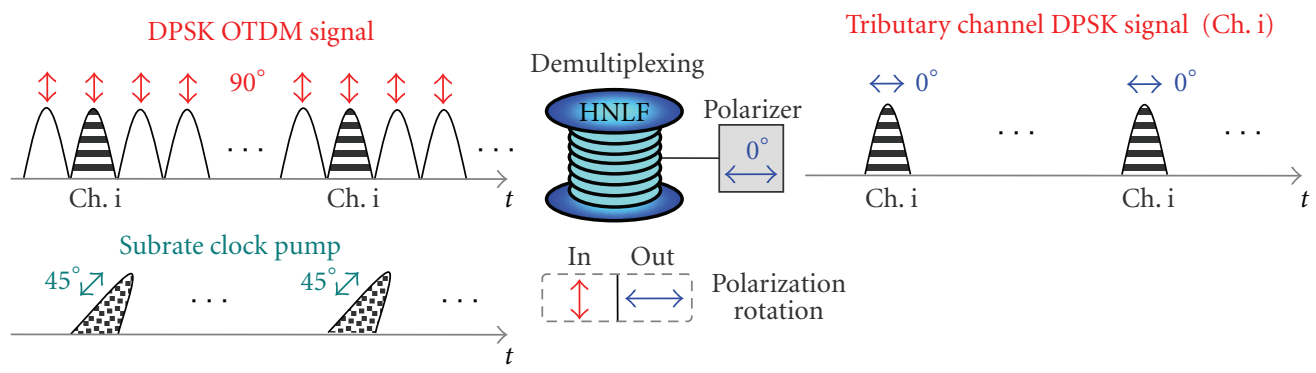

(a)
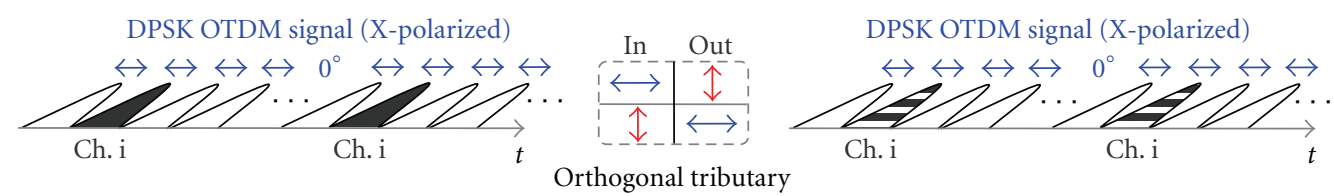

DPSK OTDM signal (Y-polarized)

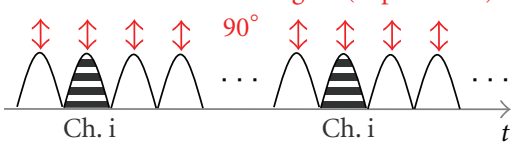

channel exchange

DPSK OTDM signal (Y-polarized)
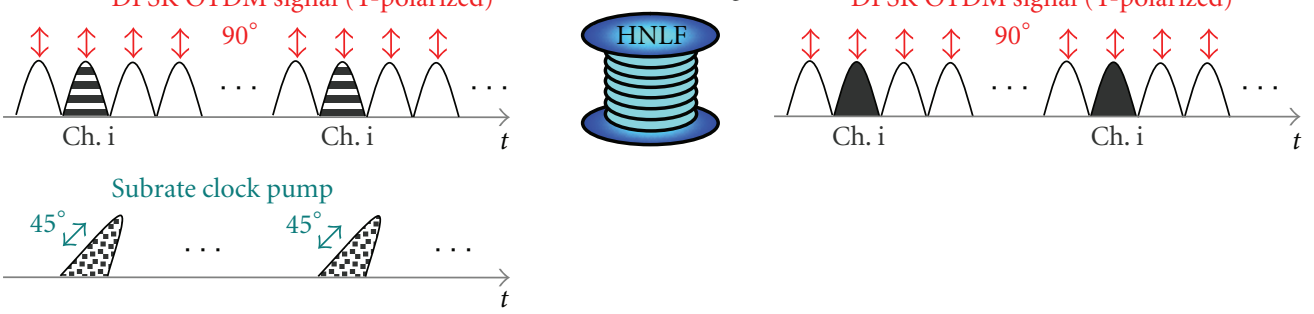

(b)

FIgURE 29: Concept and principle of Kerr effect-based (a) DPSK OTDM signal demultiplexing and (b) orthogonal tributary channel exchange of a pol-muxed DPSK OTDM signal.

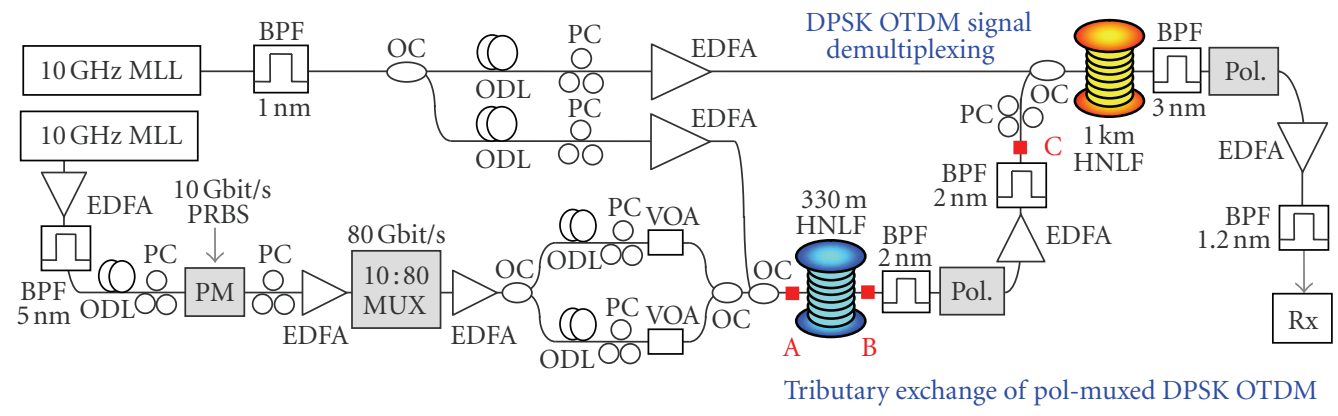

FIGURE 30: Experimental setup for the demultiplexing of a DPSK OTDM signal and the orthogonal tributary channel exchange of a polmuxed DPSK OTDM signal. HNLF: highly nonlinear fiber; MLL: mode-locked laser; EDFA: erbium-doped fiber amplifier; ODL: optical tunable delay line; BPF: band-pass filter; PC: polarization controller; PM: phase modulator; MUX: multiplexing; OC: optical coupler; VOA: variable optical attenuator; PoL.: polarizer; Rx: receiver.

demultiplexing the DPSK OTDM signal to $10 \mathrm{Gbit} / \mathrm{s}$ before the BER measurements. The polarizers (Pol.) after the HNLFs are used to separate the X-/Y-polarized data stream (80 Gbit/s DPSK OTDM) of a pol-muxed signal and extract the tributary channel (10 Gbit/s DPSK) after demultiplexing.

Figure 31 displays the eye diagrams measured by an optical sampling scope for the typical orthogonal tributary channel (Ch. 1) exchange of a $160 \mathrm{Gbit} / \mathrm{s}$ pol-muxed DPSK OTDM signal. As the $10 \mathrm{GHz}$ clock pump is time aligned to tributary channel 1 (Ch. 1) of the X- and Y-polarized DPSK OTDM signal, in the absence of the Y-polarization, as shown in Figure 31(b), Ch. 1 of the X-polarization is blocked by an
X-polarizer after the HNLF due to the $90^{\circ}$ rotation from the $\mathrm{X}$ - to the Y-polarization. When the Y-polarization is present but the $\mathrm{X}$-polarization is absent, Ch. 1 of the Y-polarization is inserted to the $\mathrm{X}$-polarization through the $90^{\circ}$ rotation from the $\mathrm{Y}$ - to the X-polarization, as shown in Figure 31(c). In the presence of both the $\mathrm{X}$ - and Y-polarizations, the tributary Ch. 1 of the Y-polarization is changed to the X-polarization, as shown in Figure 31(d). Meanwhile, the original tributary Ch. 1 of the X-polarization is also changed to the Y-polarization, as shown in Figure 31(h), resulting in the orthogonal tributary channel exchange of a pol-muxed DPSK OTDM signal. Figures 32(a) and 32(b) present other two examples of 
(a)

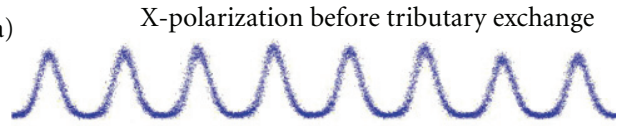

X-polarization Ch. 1 block

(b)

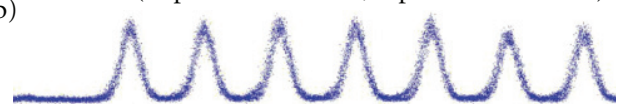

(c)

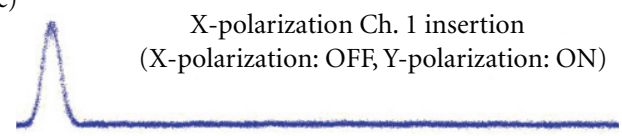

X-polarization after tributary exchange (Ch. 1)

(d)

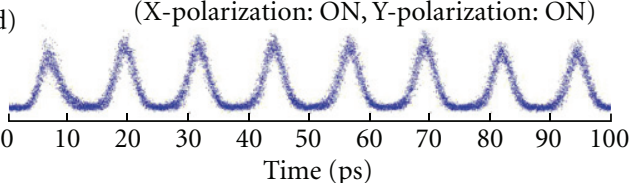

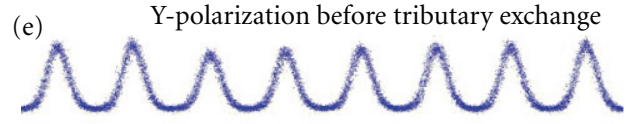

Y-polarization Ch. 1 block

(f)

(X-polarization: OFF, Y-polarization: $\mathrm{ON}$ )

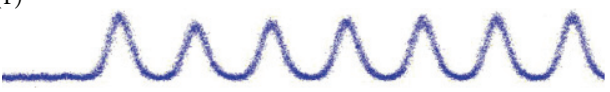

(g) Y-polarization Ch. 1 insertion

(X-polarization: ON, Y-polarization: OFF)

Y-polarization after tributary exchange (Ch. 1)

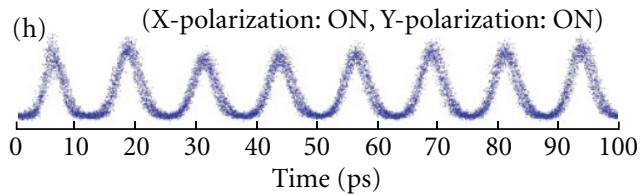

FIGURE 31: Eye diagrams of orthogonal tributary channel (Ch.1) exchange of a $160 \mathrm{Gbit} / \mathrm{s}$ pol-muxed DPSK OTDM signal.

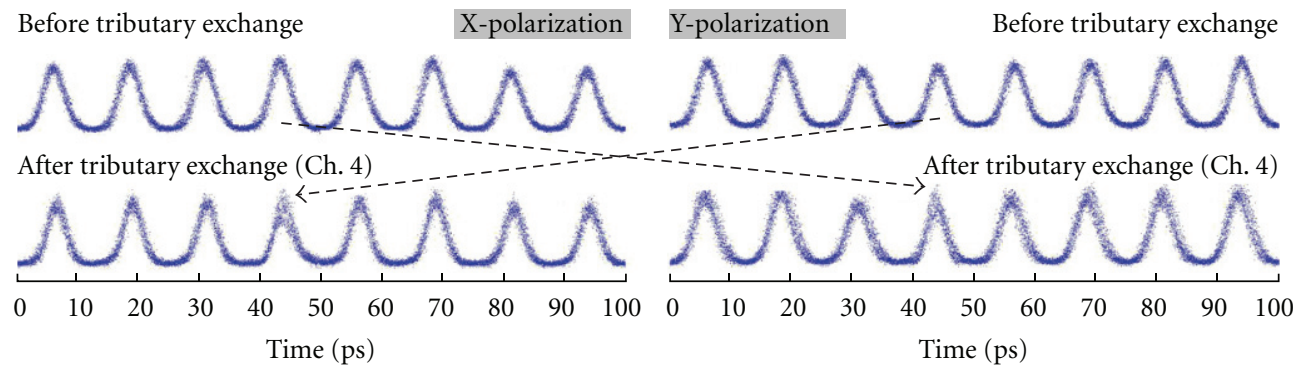

(a)

Before tributary exchange X-polarization Y-polarization Before tributary exchange
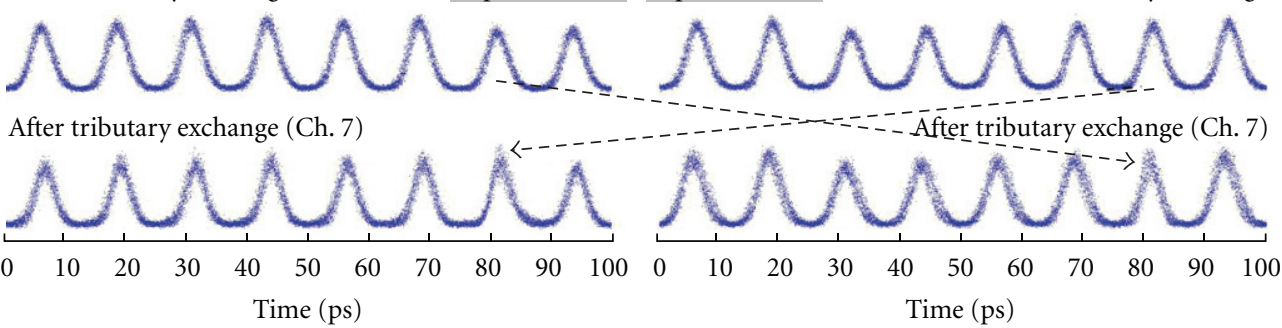

(b)

FIGURE 32: Eye diagrams of orthogonal tributary exchange of a $160 \mathrm{Gbit} / \mathrm{s}$ pol-muxed DPSK OTDM signal. (a) Ch. 4. (b) Ch. 7.

the orthogonal tributary channel (Ch. 4, Ch. 7) exchange of a $160 \mathrm{Gbit} / \mathrm{s}$ pol-muxed DPSK OTDM signal.

Figure 33 further depicts the BER performance and typical balanced eyes for the orthogonal tributary channel exchange of a $160 \mathrm{Gbit} / \mathrm{s}$ pol-muxed DPSK OTDM signal. The approximately $3.3 \mathrm{~dB}$ power penalty at a BER of $10^{-9}$ is observed during the orthogonal tributary channel exchange, which can be ascribed to the beating effect between the newly inserted signal and the original residual signal. Figure 34 plots the power penalties of the orthogonal tributary exchange for 8 tributary channels. Less than $4 \mathrm{~dB}$ power penalty at a BER of $10^{-9}$ is obtained for all 8 tributary channels with a fluctuation of $<1.5 \mathrm{~dB}$.

Remarkably, the demonstrated miscellaneous data exchange functionalities provide great potential for facilitating flexible networks. However, in practical applications, a comprehensive consideration may require some supplementary functionalities to construct a complete and independent data exchange module. Taking Figure 8 as an example (i.e., $10 \mathrm{Gbit} / \mathrm{s}$ tributary channel exchange between two $160 \mathrm{Gbit} / \mathrm{s}$ OTDM signals), all pumps and signals in the experimental setup origin from the same mode-locked laser. In real 

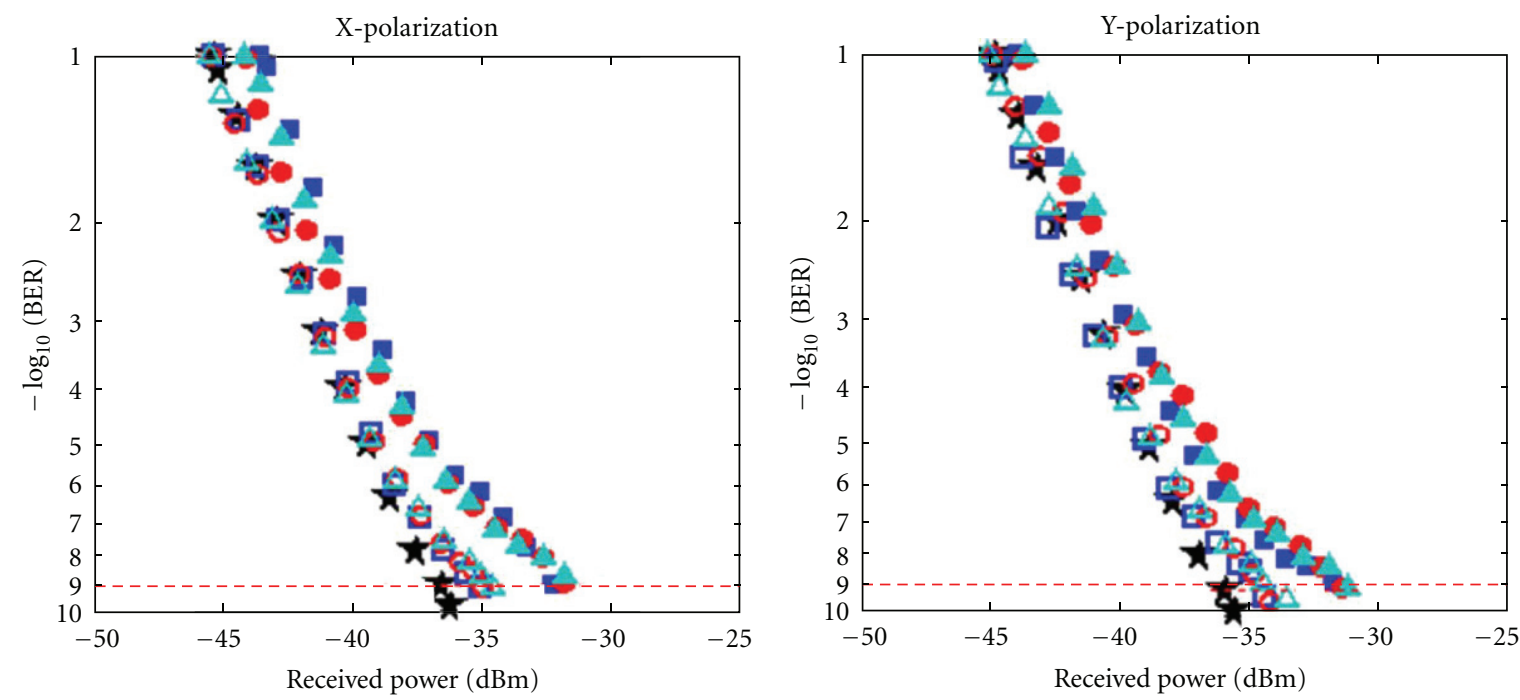
$\downarrow$ B-to-B
$\square$ Ch. 1 before exchange
O Ch. 4 before exchange
$\triangle$ Ch. 7 before exchange
Ch. 1 after exchange
- Ch. 4 after exchange
$\triangle$ Ch. 7 after exchange

\section{X-polarization}

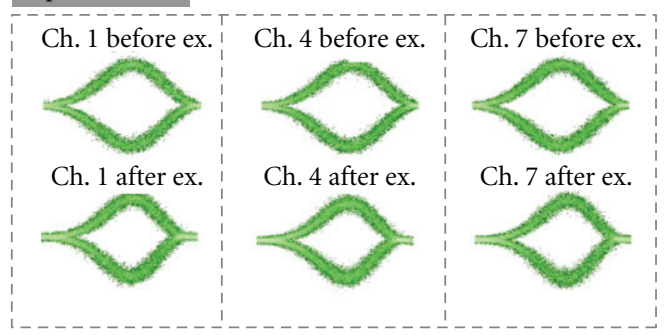

(a)

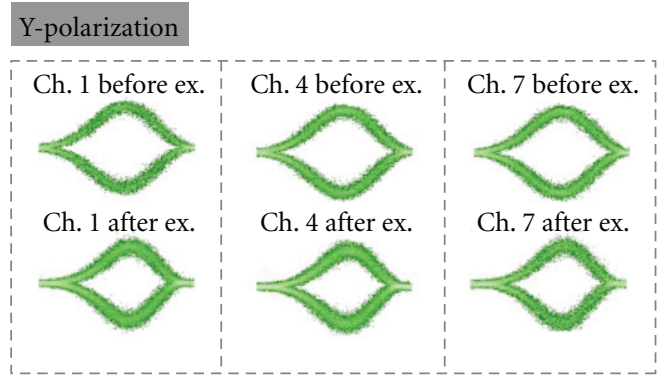

(b)

FIGURE 33: BER and balanced eyes of orthogonal tributary channel exchange of a $160 \mathrm{Gbit} / \mathrm{s}$ pol-muxed DPSK OTDM signal.

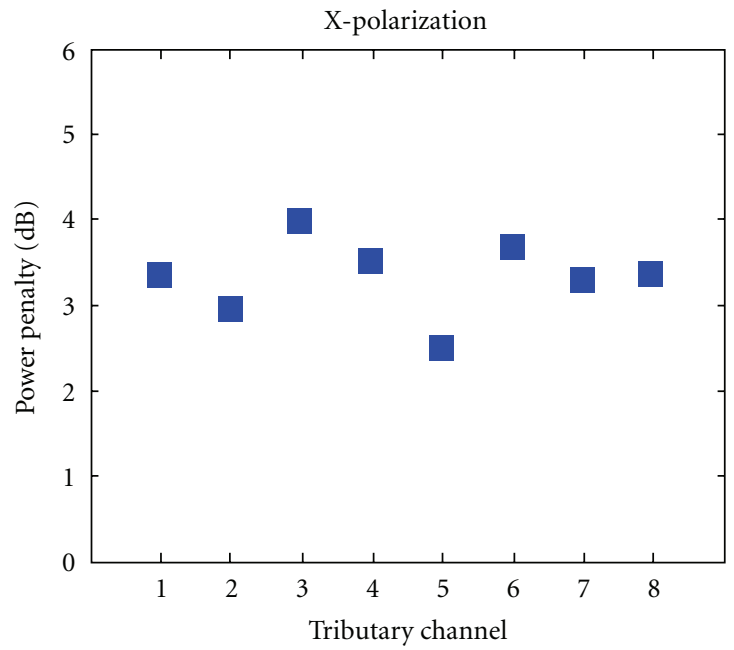

(a)

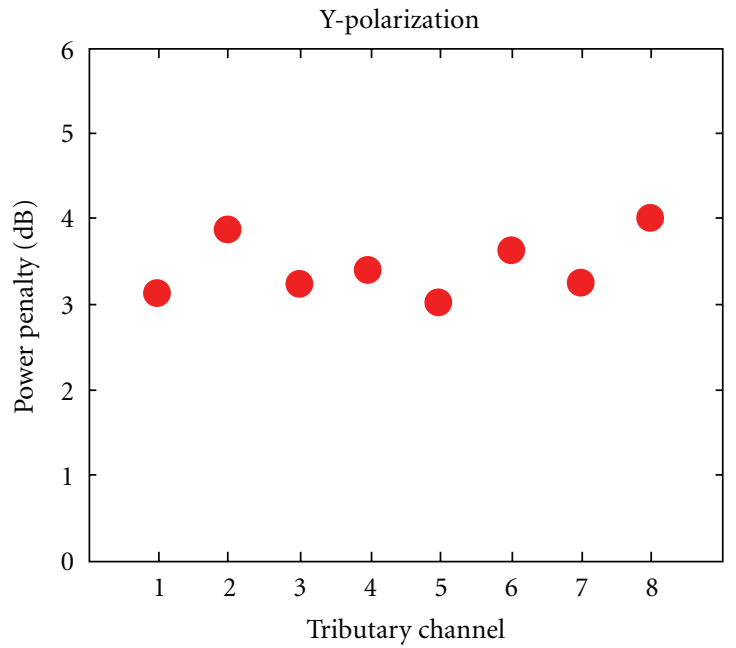

(b)

FIgURE 34: Power penalties of orthogonal tributary exchange for 8 tributary channels. 
situations, the data exchange module accepts incoming signals for tributary channel exchange assisted by locally generated pumps. The pumps and signals are usually independent with each other, and therefore a supplementary functionality of clock recovery is required for the synchronization between pumps and signals. Fortunately, various optical clock recovery methods have been developed, including the use of phase-locked loops, self-pulsating lasers, and filtering methods [70]. Optical clock recovery from DPSK signals has also been studied [71-74]. In particular, recent promising demonstrations have shown the successful synchronization and subclock recovery for ultra-high-speed OTDM signals up to $640 \mathrm{Gbit} / \mathrm{s}$ in platforms such as filter-assisted cross-phase modulation (XPM) in a semiconductor optical amplifier (SOA) $[75,76]$, sum-frequency generation (SFG) in a PPLN waveguide [77-81], and XPM in an HNLF [82-84]. As a consequence, it is possible to further develop a complete and independent data exchange module by including a synchronization and clock recovery unit.

\section{Conclusion}

We have reviewed our recent works towards robust data exchange in different degrees of freedom (wavelength, time, polarization), using various optical nonlinearities. Four kinds of optical nonlinearities, that is, cSFG/DFG in a PPLN waveguide, nondegenerate FWM in an HNLF, bidirectional degenerate FWM in an HNLF, and Kerr-induced nonlinear polarization rotation in an HNLF, are exploited to enable robust data exchange in different degrees of freedom (wavelength, time, polarization), for different modulation formats (OOK, DPSK, DQPSK, pol-muxed), and at different granularities (entire data, groups of bits, tributary channels). First, analytical solutions to the single-PPLN-based data exchange are derived showing the exchange condition. $40 \mathrm{Gbit} / \mathrm{s}$ time(groups of bits) and channel-selective data exchange between four WDM channels is implemented. $10 \mathrm{Gbit} / \mathrm{s}$ tributary channel exchange between two WDM $160 \mathrm{Gbit} / \mathrm{s}$ OTDM signals is demonstrated. Second, analytical solutions to the nondegenerate FWM-based data exchange are derived indicating the exchange condition and implying the characteristic of modulation-format transparency. Phase-transparent data exchanges (entire data) of $40 \mathrm{Gbit} / \mathrm{s}$ NRZ-DPSK signals and $100 \mathrm{Gbit} / \mathrm{s}$ RZ-DQPSK signals are demonstrated. Third, a simple approach is proposed to perform simultaneous multichannel data exchange using bidirectional degenerate FWM in an HNLF. ITU-grid-compatible four-channel $100 \mathrm{Gbit} / \mathrm{s}$ RZ-DQPSK data exchange is demonstrated. Furthermore, a reconfigurable Tbit/s network switching element is constructed using double-pass LCoS technology, together with bidirectional degenerate FWM in a single HNLF. LCoS+HNLF-based 2.3 Tbit/s (23 X 100 Gbit/s RZ-DQPSK) multifunctional grooming switch (i.e., simultaneous selective add/drop, switchable data exchange including six-channel data exchange, and power equalization) is implemented. Finally, $10 \mathrm{Gbit} / \mathrm{s}$ tributary channel exchange between two orthogonal polarizations of a $160 \mathrm{Gbit} / \mathrm{s}$ pol-muxed DPSK OTDM signal is demonstrated based on the Kerr-induced nonlinear polarization rotation. The reported theoretical and experimental results of data exchange in the wavelength, time, and polarization domains show that robust data exchange for different modulation formats and at different granularities could potentially enhance the efficiency and flexibility of optical networks.

\section{Acknowledgments}

The authors acknowledge Xiaoxia Wu, Jeng-yuan Yang, Scott R. Nuccio, Omer F. Yilmaz, Zahra Bakhtiari, Hao Huang, Xue Wang, Irfan Fazal, Yinying Xiao-Li, Yang Yue, Lin Zhang, and Robert W. Hellwarth for the helpful discussions, and the the generous support of the National Natural Science Foundation of China (NSFC) under Grant 61077051, the Program for New Century Excellent Talents in University (NCET-110182), the Natural Science Foundation of Hubei Province of China under Grant 2011CDB032, Fundamental Research Funds for the Central Universities, Huazhong University of Science and Technology (HUST;2010MS035), and the Defense Advanced Research Projects Agency (DARPA) under Contract FA8650-08-1-7820.

\section{References}

[1] J. M. Simmons, Optical Network Design and Planning, chapter 6, Springer, New York, NY, USA, 2008.

[2] K. Zhu and B. Mukherjee, "A review of traffic grooming in WDM optical networks: architectures and challenges," SPIE Optical Networks Magazine, vol. 4, no. 2, pp. 55-64, 2003.

[3] C. Caucheteur, A. Mussot, S. Bette et al., "All-fiber tunable optical delay line," Optics Express, vol. 18, no. 3, pp. 30933100, 2010.

[4] D. Dahan and G. Eisenstein, "Tunable all optical delay via slow and fast light propagation in a Raman assisted fiber optical parametric amplifier: a route to all optical buffering," Optics Express, vol. 13, no. 16, pp. 6234-6249, 2005.

[5] Y. Dai, X. Chen, Y. Okawachi et al., "1 $\mu$ s tunable delay using parametric mixing and optical phase conjugation in Si waveguides," Optics Express, vol. 17, no. 9, pp. 7004-7010, 2009.

[6] Y. Dai, Y. Okawachi, A. C. Turner-Foster, M. Lipson, A. L. Gaeta, and C. Xu, "Ultralong continuously tunable parametric delays via a cascading discrete stage," Optics Express, vol. 18, no. 1, pp. 333-339, 2010.

[7] E. Myslivets, N. Alic, S. Moro et al., "1.56- $\mu$ s continuously tunable parametric delay line for a 40-Gb/s signal," Optics Express, vol. 17, no. 14, pp. 11958-11964, 2009.

[8] S. R. Nuccio, O. F. Yilmaz, X. Wang, J. Wang, X. Wu, and A. E. Willner, "Continuously tunable $1.16 \mu$ s optical delay of 100 Gbit/s DQPSK and 50 Gbit/s DPSK signals using wavelength conversion and chromatic dispersion," Optics Letters, vol. 35, no. 11, pp. 1819-1821, 2010.

[9] S. R. Nuccio, O. F. Yilmaz, X. Wang et al., "Higher-order dispersion compensation to enable a $3.6 \mu$ s wavelength-maintaining delay of a $100 \mathrm{~Gb} / \mathrm{s}$ DQPSK signal," Optics Letters, vol. 35, no. 17, pp. 2985-2987, 2010.

[10] N. Kataoka, K. Sone, N. Wada et al., "Field trial of 640-Gbit/sthroughput, granularity-flexible optical network using packetselective ROADM prototype," Journal of Lightwave Technology, vol. 27, no. 7, pp. 825-832, 2009. 
[11] H. C. H. Mulvad, M. Galili, L. K. Oxenløwe et al., "Demonstration of $5.1 \mathrm{Tbit} / \mathrm{s}$ data capacity on a single-wavelength channel," Optics Express, vol. 18, no. 2, pp. 1438-1443, 2010.

[12] C. S. Brès, A. O. J. Wiberg, J. Coles, and S. Radic, "160-Gb/s optical time division multiplexing and multicasting in parametric amplifiers," Optics Express, vol. 16, no. 21, pp. 16609 16615, 2008.

[13] C. S. Brès, J. M. Chavez Boggio, N. Alic, and S. Radic, "1-to-40 $10-\mathrm{Gb} / \mathrm{s}$ channel multicasting and amplification in wideband parametric amplifier," IEEE Photonics Technology Letters, vol. 20, no. 16, pp. 1417-1419, 2008.

[14] C. S. Bres, N. Alic, E. Myslivets, and S. Radic, "Scalable multicasting in one-pump parametric amplifier," Journal of Lightwave Technology, vol. 27, no. 3, pp. 356-363, 2009.

[15] C. S. Brès, A. O. J. Wiberg, B. P. P. Kuo, N. Alic, and S. Radic, "Wavelength multicasting of 320-Gb/s channel in self-seeded parametric amplifier," IEEE Photonics Technology Letters, vol. 21, no. 14, pp. 1002-1004, 2009.

[16] A. Biberman, B. G. Lee, A. C. Turner-Foster et al., "Wavelength multicasting in silicon photonic nanowires," Optics Express, vol. 18, no. 17, pp. 18047-18055, 2010.

[17] H. S. Hamza and J. S. Deogun, "Wavelength-exchanging cross connects (WEX) - a new class of photonic cross-connect architectures," Journal of Lightwave Technology, vol. 24, no. 3, pp. 1101-1111, 2006.

[18] P. J. Winzer and R. J. Essiambre, "Advanced optical modulation formats," Proceedings of the IEEE, vol. 94, no. 5, pp. 952-981, 2006.

[19] P. J. Winzer and R. J. Essiambre, "Advanced modulation formats for high-capacity optical transport networks," Journal of Lightwave Technology, vol. 24, no. 12, pp. 4711-4728, 2006.

[20] G. P. Agrawal, Nonlinear Fiber Optics, Academic, San Diego, Calif, USA, 3rd edition, 2002.

[21] K. Mori, H. Takara, and M. Saruwatari, "Wavelength interchange with an optical parametric loop mirror," Electronics Letters, vol. 33, no. 6, pp. 520-522, 1997.

[22] Y. Gao, Y. Dai, C. Shu, and S. He, "Wavelength interchange of phase-shift-keying signal," IEEE Photonics Technology Letters, vol. 22, no. 11, Article ID 5443634, pp. 838-840, 2010.

[23] K. K. Y. Wong, M. E. Marhic, K. Uesaka, and L. G. Kazovsky, "Demonstration of wavelength exchange in a highly-nonlinear fiber," in Proceedings of the 27th European Conference on Optical Communication (ECOC '01), pp. 272-273, Amsterdam, The Netherlands, October 2001.

[24] K. Uesaka, K. K. Y. Wong, M. E. Marhic, and L. G. Kazovsky, "Polarization-insensitive wavelength exchange in highly-nonlinear dispersion-shifted fiber," in Proceedings of the Optical Fiber Communication Conference and Exhibit (OFC '02), pp. 563-564, March 2002, paper ThY3.

[25] K. Uesaka, K. K. Y. Wong, M. E. Marhic, and L. G. Kazovsky, "Wavelength exchange in a highly nonlinear dispersion-shifted fiber: theory and experiments," IEEE Journal on Selected Topics in Quantum Electronics, vol. 8, no. 3, pp. 560-568, 2002.

[26] R. W. L. Fung, H. K. Y. Cheung, and K. K. Y. Wong, "Widely tunable wavelength exchange in anomalous-dispersion regime," IEEE Photonics Technology Letters, vol. 19, no. 22, pp. 1846-1848, 2007.

[27] M. Shen, H. K. Y. Cheung, R. W. L. Fung, and K. K. Y. Wong, "A comprehensive study on the dynamic range of wavelength exchange and its impact on exchanged signal performance," Journal of Lightwave Technology, vol. 27, no. 14, pp. 2707-2716, 2009.
[28] H. K. Y. Cheung, R. W. L. Fung, C. H. Kwok, and K. K. Y. Wong, "All-optical packet switching by pulsed-pump wavelength exchange in a highly nonlinear dispersion-shifted fiber," in Proceedings of the Optical Fiber Communication Conference (OFC'07), 2007, paper OTuB4.

[29] M. Shen, X. Xu, T. I. Yuk, and K. K. Y. Wong, "Byte-level parametric wavelength exchange for narrow pulsewidth return-to-zero signal," IEEE Photonics Technology Letters, vol. 21, no. 21, pp. 1591-1593, 2009.

[30] C. H. Kwok, B. P. P. Kuo, and K. K. Y. Wong, "Pulsed pump wavelength exchange for high speed signal de-multiplexing," Optics Express, vol. 16, no. 15, pp. 10894-10899, 2008.

[31] M. Shen, X. Xu, T. I. Yuk, and K. K. Y. Wong, "A 160-Gb/s OTDM demultiplexer based on parametric wavelength exchange," IEEE Journal of Quantum Electronics, vol. 45, no. 11, pp. 1309-1316, 2009.

[32] A. Chowdhury, S. C. Hagness, and L. McCaughan, "Simultaneous optical wavelength interchange with a two-dimensional second-order nonlinear photonic crystal," Optics Letters, vol. 25, no. 11, pp. 832-834, 2000.

[33] A. Chowdhury, C. Staus, B. F. Boland, T. F. Kuech, and L. McCaughan, "Experimental demonstration of 1535-1555 nm simultaneous optical wavelength interchange with a nonlinear photonic crystal," Optics Letters, vol. 26, no. 17, pp. 1353-1355, 2001.

[34] J. Wang and Q. Sun, "Theoretical analysis of power swapping in quadratic nonlinear medium," Applied Physics Letters, vol. 96, no. 8, Article ID 081108, 2010.

[35] J. Wang, S. R. Nuccio, X. Wu et al., "40-Gbit/s optical data exchange between WDM channels using second-order nonlinearities in PPLN waveguides," in Proceedings of the Conference on Nonlinear Optics (NLO '09), Honolulu, Hawaii, USA, 2009, PDPA1.

[36] J. Wang, S. Nuccio, X. Wu et al., "40 Gbit/s optical data exchange between wavelength-division-multiplexed channels using a periodically poled lithium niobate waveguide," Optics Letters, vol. 35, no. 7, pp. 1067-1069, 2010.

[37] J. Wang, Z. Bakhtiari, Y. Xiao-Li et al., "Experimental demonstration of data traffic grooming of a single 10-Gbit/s TDM tributary channel between two 160-Gbit/s WDM channels," in Proceedings of the Optical Fiber Communication Conference (OFC'10), San Diego, Calif, USA, 2010, paper OWF1.

[38] J. Wang, Z. Bakhtiari, O. F. Yilmaz, S. R. Nuccio, X. Wu, and A. E. Willner, "10 Gbit/s tributary channel exchange of 160 Gbit/s signals using periodically poled lithium niobate," Optics Letters, vol. 36, no. 5, pp. 630-632, 2011.

[39] J. Wang, Z. Bakhtiari, Y. Xiao-Li et al., "Phase-transparent optical data exchange of 40-Gbit/s DPSK signals using fourwave-mixing in a highly nonlinear fiber," in Proceedings of the Optical Fiber Communication Conference (OFC '10), San Diego, Calif, USA, 2010, paper OMT6.

[40] J. Wang, Z. Bakhtiari, S. R. Nuccio, O. F. Yilmaz, X. Wu, and A. E. Willner, "Phase-transparent optical data exchange of 40 Gbit/s differential phase-shift keying signals," Optics Letters, vol. 35, no. 17, pp. 2979-2981, 2010.

[41] J. Wang, S. R. Nuccio, H. Huang et al., "Demonstration of 100-Gbit/s DQPSK data exchange between two different wavelength channels using parametric depletion in a highly nonlinear fiber," in Proceedings of the European Conference on Optical Communications (ECOC '10), Torino, Italy, 2010, paper Mo.1.A.4.

[42] J. Wang, S. R. Nuccio, H. Huang, X. Wang, J. Y. Yang, and A. E. Willner, "Optical data exchange of 100-Gbit/s DQPSK signals," Optics Express, vol. 18, no. 23, pp. 23740-23745, 2010. 
[43] J. Wang, H. Huang, X. Wang, J.-Y. Yang, and A. E. Willner, "Optical phase-transparent data grooming exchange of multichannel 100-Gbit/s RZ-DQPSK signals," in Proceedings of the 23rd IEEE Photonics Society Annual Meeting, Denver, Colo, USA, 2010, paper WN2.

[44] J. Wang, H. Huang, X. Wang, J.-Y. Yang, and A. E. Willner, "Multi-channel 100-Gbit/s DQPSK data exchange using bidirectional degenerate four-wave mixing," Optics Express, vol. 19, no. 4, pp. 3332-3338, 2011.

[45] J. Wang, H. Huang, X. Wang et al., "2.3-Tbit/s (23X100Gbit/s) RZ-DQPSK grooming switch (simultaneous add/drop, data exchange and equalization) using double-pass LCoS and bidirectional HNLF," in Proceedings of the Optical Fiber Communication Conference (OFC '11), Los Angeles, Calif, USA, 2011, paper OTuE2.

[46] J. Wang, H. Huang, X. Wang, J.-Y. Yang, and A. E. Willner, "Reconfigurable 2.3-Tbit/s DQPSK simultaneous add/drop, data exchange and equalization using double-pass LCoS and bidirectional HNLF," Optics Express, vol. 19, no. 19, pp. 1824618252, 2011.

[47] J. Wang, Z. Bakhtiari, Y. Xiao-Li et al., "Experimental demonstration of data traffic grooming of a single 10-Gbit/s TDM tributary channel between two 160-Gbit/s WDM channels," in Proceedings of the Conference on Lasers and Electro-Optics (CLEO '01), San Jose, Calif, USA, March 2010, paper CFJ5.

[48] J. Wang, O. F. Yilmaz, S. R. Nuccio, X. Wu, and A. E. Willner, "Orthogonal tributary channel exchange of 160-Gbit/s polmuxed DPSK signal," Optics Express, vol. 18, no. 16, pp. 16995-17008, 2010.

[49] A. E. Willner, O. F. Yilmaz, J. Wang et al., "Optically efficient nonlinear signal processing," IEEE Journal on Selected Topics in Quantum Electronics, vol. 17, no. 2, pp. 320-332, 2011.

[50] K. R. Parameswaran, M. Fujimura, M. H. Chou, and M. M. Fejer, "Low-power all-optical gate based on sum frequency mixing in APE waveguides in PPLN," IEEE Photonics Technology Letters, vol. 12, no. 6, pp. 654-656, 2000.

[51] J. Wang, J. Sun, and Q. Sun, "Experimental observation of a $1.5 \mu \mathrm{m}$ band wavelength conversion and logic NOT gate at 40 Gbit/s based on sum-frequency generation," Optics Letters, vol. 31, no. 11, pp. 1711-1713, 2006.

[52] J. Wang, J. Sun, and Q. Sun, "Single-PPLN-based simultaneous half-adder, half-subtracter, and or logic gate: proposal and simulation," Optics Express, vol. 15, no. 4, pp. 1690-1699, 2007.

[53] X. X. Wu, A. Bogoni, H. Huang et al., "Reconfigurable 40Gbit/s tributary selection from a $640-\mathrm{Gbit} / \mathrm{s}$ signal using NOLM-based cascaded demultiplexing," in Proceedings of the European Conference on Optical Communications (ECOC'10), Torino, Italy, 2010, paper P3.01.

[54] J. Huang, J. R. Kurz, C. Langrock, A. M. Schober, and M. M. Fejer, "Quasi-group-velocity matching using integrated-optic structures," Optics Letters, vol. 29, no. 21, pp. 2482-2484, 2004.

[55] A. Kobyakov, M. Sauer, and D. Chowdhury, "Stimulated Brillouin scattering in optical fibers," Advances in Optics and Photonics, vol. 2, no. 1, pp. 1-59, 2010.

[56] K. K. Y. Wong, K. Shimizu, K. Uesaka, G. Kalogerakis, M. E. Marhic, and L. G. Kazovsky, "Continuous-wave fiber optical parametric amplifier with $60-\mathrm{dB}$ gain using a novel twosegment design," IEEE Photonics Technology Letters, vol. 15, no. 12, pp. 1707-1709, 2003.

[57] J. M. Chavez Boggio, J. D. Marconi, and H. L. Fragnito, "Experimental and numerical investigation of the SBS-threshold increase in an optical fiber by applying strain distributions," Journal of Lightwave Technology, vol. 23, no. 11, pp. 3808-3814, 2005.

[58] J. Hansryd, F. Dross, M. Westlund, P. A. Andrekson, and S. N. Knudsen, "Increase of the SBS threshold in a short highly nonlinear fiber by applying a temperature distribution," Journal of Lightwave Technology, vol. 19, no. 11, pp. 1691-1697, 2001.

[59] A. Liu, "Suppressing stimulated Brillouin scattering in fiber amplifiers using nonuniform fiber and temperature gradient," Optics Express, vol. 15, no. 3, pp. 977-984, 2007.

[60] A. Kobyakov, S. Kumar, D. Q. Chowdhury et al., "Design concept for optical fibers with enhanced SBS threshold," Optics Express, vol. 13, no. 14, pp. 5338-5346, 2005.

[61] S. R. Bickham, A. Kobyakov, and S. Li, "Nonlinear optical fibers with increased SBS thresholds," in Proceedings of the Optical Fiber Communication Conference and Exposition and The National Fiber Optic Engineers Conference, Technical Digest (CD), Optical Society of America, 2006, paper OTuA3.

[62] T. Sakamoto, T. Matsui, K. Shiraki, and T. Kurashima, "SBS suppressed fiber with hole-assisted structure," Journal of Lightwave Technology, vol. 27, no. 20, pp. 4401-4406, 2009.

[63] J. H. Lee, T. Nagashima, T. Hasegawa, S. Ohara, N. Sugimoto, and K. Kikuchi, "Bismuth-oxide-based nonlinear fiber with a high SBS threshold and its application to four-wave-mixing wavelength conversion using a pure continuous-wave pump," Journal of Lightwave Technology, vol. 24, no. 1, pp. 22-27, 2006.

[64] T. Tsuzaki, T. Miyamoto, T. Okuno et al., "Impact of double Rayleigh backscattering in discrete fiber Raman amplifiers employing highly nonlinear fiber," in Proceedings of the OSA Topical Meeting Optical Amplifiers and Their Applications (OAA '95), Vancouver, Canada, 2002, paper OWA2.

[65] M. A. F. Roelens, S. Frisken, J. A. Bolger et al., "Dispersion trimming in a reconfigurable wavelength selective switch," Journal of Lightwave Technology, vol. 26, no. 1, pp. 73-78, 2008.

[66] M. A. F. Roelens, J. A. Bolger, D. Williams, and B. J. Eggleton, "Multi-wavelength synchronous pulse burst generation with a wavelength selective switch," Optics Express, vol. 16, no. 14, pp. 10152-10157, 2008.

[67] J. Suzuki, K. Taira, Y. Fukuchi, Y. Ozeki, T. Tanemura, and K. Kikuchi, "All-optical time-division add-drop multiplexer using optical fibre Kerr shutter," Electronics Letters, vol. 40, no. 7, pp. 445-446, 2004.

[68] V. Marembert, K. Schulze, C. Schubert et al., "Investigations of fiber Kerr switch: nonlinear phase shift measurements and optical time-division demultiplexing of $320 \mathrm{Gbit} / \mathrm{s}$ DPSK signals," in Proceedings of the Conference on Lasers and ElectroOptics (CLEO '05), May 2005, paper CWK7.

[69] E. J. M. Verdurmen, A. M. J. Koonen, and H. De Waardt, "Time domain add-drop multiplexing for RZ-DPSK OTDM signals," Optics Express, vol. 14, no. 12, pp. 5114-5120, 2006.

[70] T. von Lerber, S. Honkanen, A. Tervonen, H. Ludvigsen, and F. Küppers, "Optical clock recovery methods: review (invited)," Optical Fiber Technology, vol. 15, no. 4, pp. 363-372, 2009.

[71] G. Contestabile, M. Presi, N. Calabretta, and E. Ciaramella, "All-optical clock recovery for NRZ-DPSK signals," IEEE Photonics Technology Letters, vol. 18, no. 23, pp. 2544-2546, 2006.

[72] S. Fu, M. Tang, W. D. Zhong, Y. J. Wen, and P. Shum, "Alloptical NRZ-DPSK clock recovery using chromatic-dispersion-induced clock tone," IEEE Photonics Technology Letters, vol. 19, no. 12, pp. 925-927, 2007.

[73] G. Contestabile, R. Proietti, N. Calabretta, M. Presi, A. D'Errico, and E. Ciaramella, "Simultaneous demodulation 
and clock-recovery of 40-gb/s nrz-dpsk signals using a multiwavelength gaussian filter," IEEE Photonics Technology Letters, vol. 20, no. 10, pp. 791-793, 2008.

[74] X. Tang, J. C. Cartledge, A. Shen, A. Akrout, and G. H. Duan, "Low-timing-jitter all-optical clock recovery for $40 \mathrm{Gbits} / \mathrm{s}$ RZ-DPSK and NRZ-DPSK signals using a passively modelocked quantum-dot Fabry-Perot semiconductor laser," Optics Letters, vol. 34, no. 7, pp. 899-901, 2009.

[75] E. Tangdiongga, H. C. H. Mulvad, H. de Waardt, G. D. Khoe, A. M. J. Koonen, and H. J. S. Dorren, "SOA-based clock recovery and demultiplexing in a lab trial of $640 \mathrm{~Gb} / \mathrm{s}$ OTDM transmission over 50-km fibre link," in Proceedings of the European Conference on Optical Communication (ECOC '07), 2007, postdeadline paper PD 1.2.

[76] H. C. H. Mulvad, E. Tangdiongga, H. De Waardt, and H. J. S. Dorren, "40GHz clock recovery from 640Gbit/s OTDM signal using SOA-based phase comparator," Electronics Letters, vol. 44, no. 2, pp. 146-148, 2008.

[77] F. G. Agis, C. Ware, and D. Erasme, "Clock synchronization and sub-clock extraction of optical signals at high rates using an opto-electronic PLL based on three-wave mixing in PPLN," in Proceedings of the 9th International Conference on Transparent Optical Networks (ICTON '07), pp. 232-233, July 2007, paper We.D2.4.

[78] L. K. Oxenløwe, F. Gomez Agis, C. Ware et al., "640Gbit/s clock recovery using periodically poled lithium niobate," Electronics Letters, vol. 44, no. 5, pp. 370-372, 2008.

[79] C. Ware, L. K. Oxenløwe, F. G. Agis et al., "320 Gbps to 10 $\mathrm{GHz}$ sub-clock recovery using a PPLN-based opto-electronic phase-locked loop," Optics Express, vol. 16, no. 7, pp. 50075012, 2008.

[80] L. K. Oxenløwe, F. Gómez-Agis, C. Ware et al., “640-Gbit/s data transmission and clock recovery using an ultrafast periodically poled lithium niobate device," Journal of Lightwave Technology, vol. 27, no. 3, pp. 205-213, 2009.

[81] L. K. Oxenløwe, M. Galili, H. C. Hansen Mulvad et al., "Nonlinear optical signal processing for Tbit/s Ethernet applications," International Journal of Optics, vol. 2012, Article ID 573843, 14 pages, 2012.

[82] A. O. J. Wiberg, C. Brès, B. P.-P. Kuo, E. Myslivets, N. Alic, and S. Radic, "320 Gbps DPSK transmitter and selftracked receiver based on four-wave mixing," in Proceedings of the Optical Fiber Communication Conference, OSA Technical Digest (CD), Optical Society of America, 2011, paper OWL1.

[83] B. P.-P. Kuo, A. O. J. Wiberg, C.-S. Brès, N. Alic, and S. Radic, "Ultrafast clock recovery and sampling by single parametric device," IEEE Photonics Technology Letters, vol. 23, no. 3, pp. 191-193, 2011.

[84] A. O. J. Wiberg, B. P.-P. Kuo, C.-S. Brès, N. Alic, and S. Radic, " $640-\mathrm{Gb} / \mathrm{s}$ transmitter and self-tracked demultiplexing receiver using single parametric gate," IEEE Photonics Technology Letters, vol. 23, no. 8, pp. 507-509, 2011. 

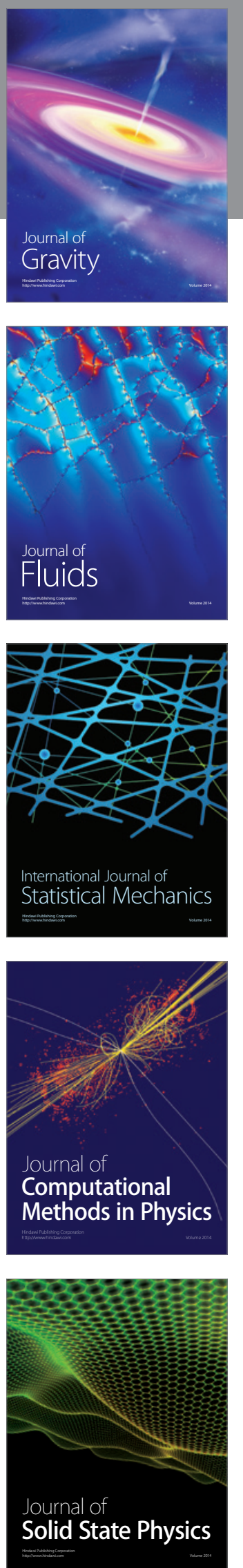

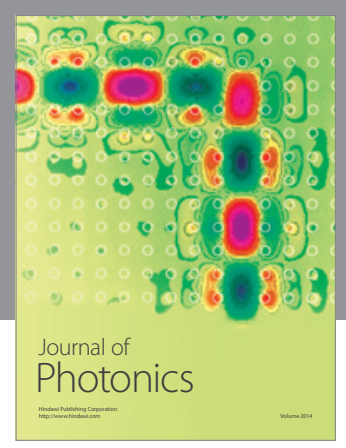

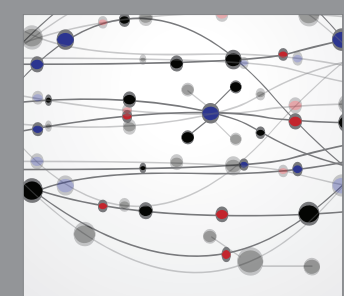

The Scientific World Journal
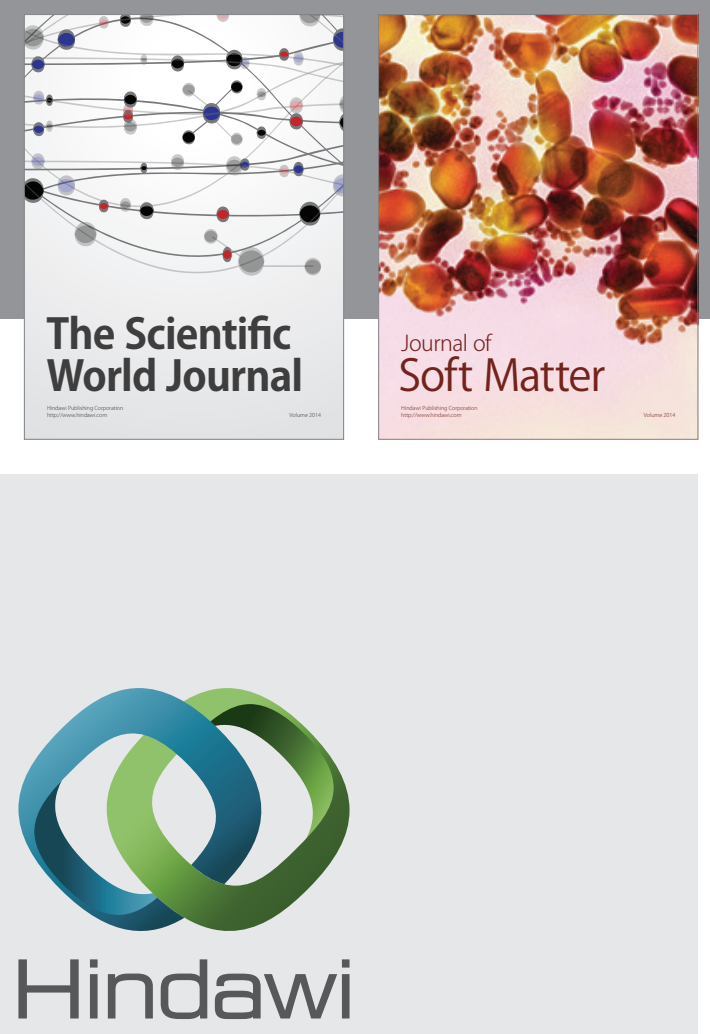

Submit your manuscripts at

http://www.hindawi.com
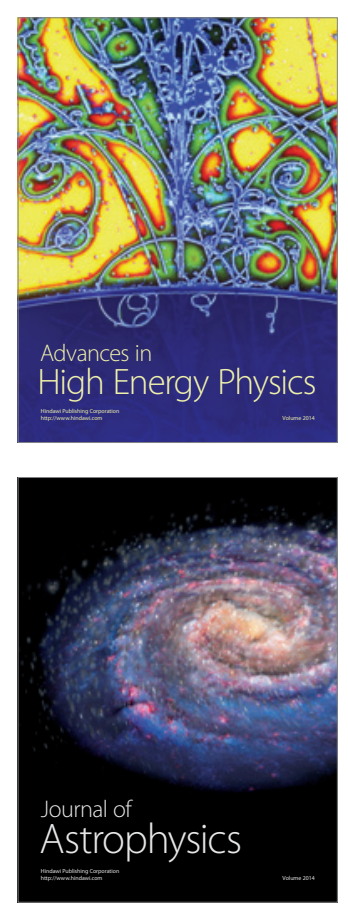
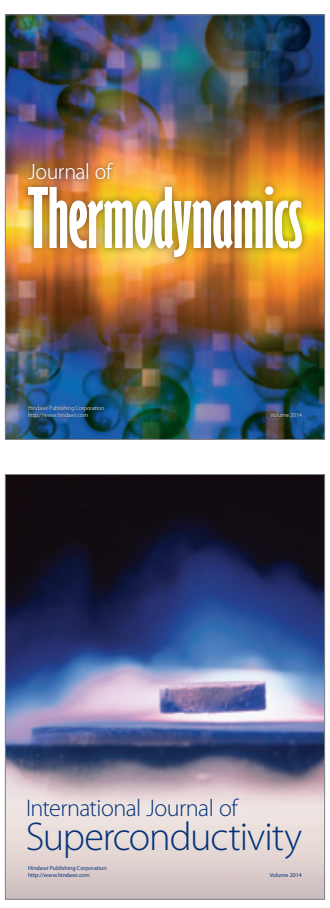
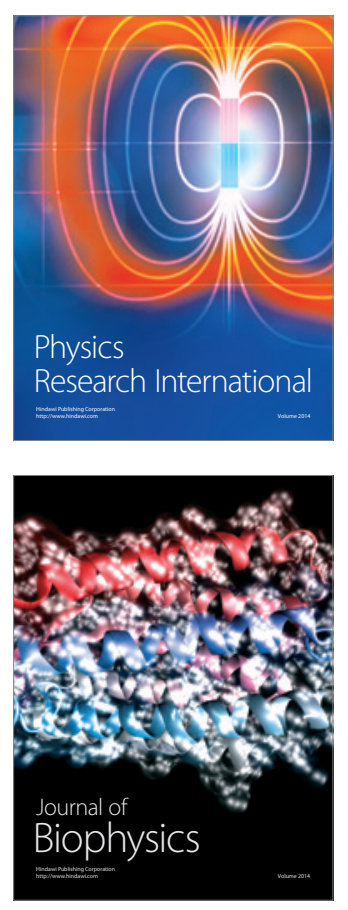
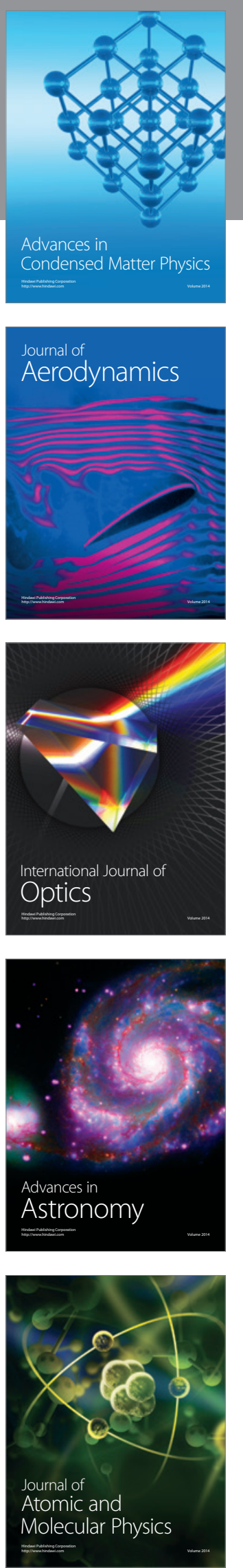